\title{
Tracing shock type with chemical diagnostics
}

\section{An application to L1157}

\author{
T. A. James ${ }^{1}$, S. Viti ${ }^{1}$, J. Holdship ${ }^{1}$, and I. Jiménez-Serra ${ }^{2}$ \\ 1 Department of Physics and Astronomy, University College London, Gower Street, London WC1E 6BT, UK \\ e-mail: tjames@star.ucl.ac.uk; t.james.17@ucl.ac.uk \\ 2 Centro de Astrobiologia (CSIC, INTA), Ctra. de Ajalvir, km. 4, Torrejón de Ardoz 28850, Madrid, Spain
}

Received 20 August 2019 / Accepted 4 December 2019

\begin{abstract}
Aims. The physical structure of a shock wave may take a form unique to its shock type, implying that the chemistry of each shock type is unique as well. We aim to investigate the different chemistries of J-type and C-type shocks in order to identify unique molecular tracers of both shock types. We apply these diagnostics to the protostellar outflow L1157 to establish whether the B2 clump could host shocks exhibiting type-specific behaviour. Of particular interest is the L1157-B2 clump, which has been shown to exhibit bright emission in S-bearing species and HNCO.

Methods. We simulate, using a parameterised approach, a planar, steady-state J-type shock wave using UCLCHEM. We compute a grid of models using both C-type and J-type shock models to determine the chemical abundance of shock-tracing species as a function of distance through the shock and apply it to the L1157 outflow. We focus on known shock-tracing molecules such as $\mathrm{H}_{2} \mathrm{O}, \mathrm{HCN}$, and $\mathrm{CH}_{3} \mathrm{OH}$.

Results. We find that a range of molecules including $\mathrm{H}_{2} \mathrm{O}$ and $\mathrm{HCN}$ have unique behaviour specific to a J-type shock, but that such differences in behaviour are only evident at low $v_{\mathrm{s}}$ and low $n_{\mathrm{H}}$. We find that $\mathrm{CH}_{3} \mathrm{OH}$ is enhanced by shocks and is a reliable probe of the pre-shock gas density. However, we find no difference between its gas-phase abundance in C-type and J-type shocks. Finally, from our application to L1157, we find that the fractional abundances within the B2 region are consistent with both C-type and J-type shock emission.
\end{abstract}

Key words. astrochemistry - evolution - ISM: individual objects: L1157 - ISM: molecules - stars: protostars

\section{Introduction}

Astrophysical shocks represent prominent catalysts for chemical evolution in the interstellar medium (ISM). The low signal-speed within the ambient ISM leads to a variety of different astrophysical events driving supersonic flows that form shocks, from cloudcloud collisions (e.g. Gidalevich 1966) to bipolar outflows emanating from protostellar objects (e.g. Snell et al. 1980; Shu et al. 1991; Zhang \& Zheng 1997). The different ambient gas conditions that a supersonic flow can be driven into leads to the production of different shock types. Draine (1980) initially defined two shock types, $\mathrm{C}$ (continuous) type shock and $\mathrm{J}$ (jump) type shock, with subsequent computational work by Chièze et al. (1998) and Flower et al. (2003a) defining a third, CJ (mixed) type shock.

Unlike C-type shocks, which typically arise in regions with a magnetic field and low degree of fractional-ionisation, J-type shocks arise in regions whereby only a negligible magnetic field is present (Draine 1980). The negligible magnetic field within a J-type shock has further consequences in that it does not act to limit the compression through the shock, thus allowing a higher peak temperature to be reached within the shock-front (relative to a C-type shock). Owing to this, J-type shocks are thought to exhibit far more destructive chemistry than a C-type shock counterpart. An analytic description of a C-type shock therefore requires equations of magnetohydrodynamics (MHD) and multiple fluid components, whilst J-type shocks can be described by hydrodynamics equations and a single fluid alone.
Typically, such descriptions are implemented in MHD codes such as mhd_vode (Flower \& Des ForÉts 2015). However, such approaches to modelling incur a large amount of computational expense, necessitating compromises in the complexity and size of the chemical network used. By using a parameterised form of the physical structure of the shock, as Jiménez-Serra et al. (2008) did with their C-type shock parameterisation, it is possible to preserve an approximation of the shock structure whilst significantly reducing computational complexity, thus allowing the computation of far more complex chemistry.

This is particularly important owing to the complex chemistry that is influenced by shocks. In particular, shocks can drive chemical reactions that would otherwise be highly unlikely to occur under quiescent ISM conditions. For example, the reaction $\mathrm{O}+\mathrm{H}_{2} \longrightarrow \mathrm{OH}+\mathrm{H}$ has an activation barrier of $\approx 1 \mathrm{eV}$ and would therefore require temperatures $>1000 \mathrm{~K}$, which are easily achievable within shocks, to initiate (Baulch et al. 1992; van Dishoeck et al. 2013; Williams \& Viti 2013).

It is through such reactions that the axiom of unique chemistry as a diagnostic of prior physical events is drawn. Further reinforcing this axiom is interstellar chemistry's high density and temperature dependence, thus rendering the composition of the ISM highly sensitive to dynamical environmental effects. Shocks are ubiquitous sources of such change within the ISM, and therefore represent prominent sources of chemical enrichment in early star-forming environments. 
Observations of shocked regions allow effective probes of the shock chemistry. Recent high-resolution spectroscopy programmes such as ASAI (Lefloch et al. 2018), CHESS (Lefloch et al. 2010), and WISH (van Dishoeck et al. 2010) permit unprecedented insight into not just early stages of star formation, but also the violent events that initially drive shocks into these regions. The bipolar outflow in L1157 (Umemoto et al. 1992) is an example of a prototypical protostellar outflow observed during these programmes. Observations of outflows cannot, however, provide insight into either the physical or subsequent chemical evolution of the shock through time, instead only capturing a static snapshot of the conditions. Modelling shock-induced chemistry is therefore one of the only methods of following the evolution of an inherently time-dependent chemical process in astrophysics.

The role that dust grains play in interstellar chemistry is also of paramount importance. Molecules in the gas-phase may freeze on to the surface of dust grains, thereby depleting their gas-phase abundance by changing state. Processes such as successive hydrogenation on dust grains are thought to be the mechanism responsible for such complex organic molecule formation as $\mathrm{CH}_{3} \mathrm{OH}$ (Tielens \& Whittet 1997; Fuchs et al. 2009). Importantly this method also presents a viable solution to the cold gasphase abundance problem whereby molecules are observed in the gas phase at temperatures well below their gas-phase formation temperature. Under the influence of a sputtering, graingrain collision or desorption event (thermal or non-thermal), the molecule may be released from the surface of the dust-grain directly into the gas phase. This complex interplay between the gas-phase and dust-grain chemistry essentially chemically couples the two phases. It is therefore vitally important when modelling interstellar chemistry that both gas-phase and dust-grain reactions included within the reaction network are accurate and comprehensive for the relevant molecules.

In practice, the only way one can hope to distinguish between the two types of shock is to systematically determine the effects of each shock type and hence compare the resultant chemical distinctions. Our goal in this paper is to identify molecular tracers of a J-type shock by using such a technique and apply it to a shocked region of L1157 thought to be exhibiting signatures of both C-type and J-type shock behaviour. We therefore make extensive use of the C-type shock module, based on JiménezSerra et al. (2008), that is already implemented in UCLCHEM (Holdship et al. 2017). To that goal, we present in Sect. 2 an overview of L1157. We present in Sect. 3 a parameterised model of a J-type shock built for the astrochemical code UCLCHEM. In conjunction with the pre-existing C-type shock model based upon Jiménez-Serra et al. (2008) we investigate in Sect. 4 the chemical distinctions between J-type and C-type shocks to identify unique chemical tracers of both shock types. Section 5 applies these results by comparing them to enhanced abundances with shocked regions of the L1157 outflow.

\section{L1157}

At 250 pc (Looney et al. 2007), L1157 is a nearby region that comprises a central class- 0 protostar, L1157-mm, that in turn drives a bipolar outflow. The observed outflow produces a redshifted lobe to the North and a blue-shifted lobe to the South that are aligned with the protostar's rotation axis. A degree of symmetry is observed in these lobes, however the geometry of lobe sub-structure indicates the presence of an underlying precessing jet (Vasta et al. 2012). This precession allows periodic ejection events to create complex structures enhanced by shocks (Gueth



Fig. 1. Spitzer/IRAC $8 \mu \mathrm{m}$ image of the L1157 outflow. (Podio et al. 2016). Shown as black squares are the shock events B0, B1 and B2. The class-0 protostar L1157- $\mathrm{mm}$ that drives the outflow is also labelled. The black line overplotted is the precession model thought to be responsible for the creation of the observed knots. As is visible here, B2 is far less intense in emission than B0/B1.

et al. 1996). The Southern lobe hosts two intriguing examples of such shock events: the clumps B1 and B2, which are themselves located within larger cavities C1 and C2. As a result, L1157 is considered to be one of the best laboratories for astrochemistry (Umemoto et al. 1992; Bachiller et al. 2001).

Figure 1 shows Spitzer/IRAC $8 \mu$ m observations by Podio et al. (2016). Labelled are the knots B0, B1 and B2 alongside the central driving protostar L1157-mm and the proposed precession model from Podio et al. (2016).

It has since been found that B1 and B2 themselves host low-velocity clumps. Benedettini et al. (2007), using PdB interferometric observations, showed that nine clumps exist within the B1 and B2 structure, thus giving rise to even further complexity within the Southern lobe. This substructure is thought to arise from L1157-mm's precession, which creates complex knots driven by shock-activity produced by the host outflow.

\section{1. $L 1157-B 1$}

B1 is the brightest clump within the L1157 region and thus the subject of significant study. It is warm and young, exhibiting kinetic temperatures between $T \approx 80-100 \mathrm{~K}$ and age $t \approx$ 1000 years. In comparison B2 is colder and older with $T \approx$ 20-60 K and $t \approx 4000$ years (Tafalla \& Bachiller 1995; Gueth et al. 1996). Viti et al. (2011) first showed, with confirmation by Benedettini et al. (2012), that B1 is likely produced by a non-dissociative, C-type shock with pre-shock density $n_{\mathrm{H}} \geq$ $10^{4} \mathrm{~cm}^{-3}$ and $v_{\mathrm{s}} \approx 40 \mathrm{~km} \mathrm{~s}^{-1}$, leading to a maximum obtainable temperature of $\sim 4000 \mathrm{~K}$.

\section{2. $L 1157-B 2$}

Being less intense in most emission lines, $\mathrm{B} 2$ has been subject to far less study. B2 is, however, brighter than B1 in most sulphurbearing species as well as HNCO (Tafalla \& Bachiller 1995; 
T. A. James et al.: Tracing shock type with chemical diagnostics

Table 1. Abundances $\chi$ of known shock enhanced molecules and their enhancement factors $f$ (relative to $\chi(0)$ ) in the two L1157 knots B1 and B2.

\begin{tabular}{lllllll}
\hline \hline Molecule & $\chi(0)$ & $\chi(\mathrm{B} 1)$ & $\chi(\mathrm{B} 2)$ & $f(\mathrm{~B} 1)$ & $f(\mathrm{~B} 2)$ & Reference \\
\hline $\mathrm{CH}_{3} \mathrm{OH}$ & $4.5 \times 10^{-8}$ & $0.4-1.9 \times 10^{-5}$ & $2.2 \times 10^{-5}$ & $300-400$ & 500 & $(1)$ \\
$\mathrm{HCN}$ & $3.6 \times 10^{-9}$ & $3.3 \times 10^{-7}$ & $5.5 \times 10^{-7}$ & 90 & 150 & $(1)$ \\
$\mathrm{SO}$ & $\sim 5.0 \times 10^{-9}$ & $2.0-3.0 \times 10^{-7}$ & $2.0-5.0 \times 10^{-7}$ & $50-70$ & $60-100$ & $(1)$ \\
$\mathrm{SO}_{2}$ & $\lesssim 3.0 \times 10^{-8}$ & $2.1 \times 10^{-7}$ & $5.7 \times 10^{-7}$ & $\sim 8$ & $\sim 20$ & $(1)$ \\
$\mathrm{H}_{2} \mathrm{O}$ & $(\ldots)$ & $1 \times 10^{-4}$ & $1 \times 10^{-6}$ & $\ldots)$ & $(\ldots)$ & $(2)$ \\
$\mathrm{HNCO}$ & $0.3-1.2 \times 10^{-9}$ & $4.3-17.9 \times 10^{-9}$ & $25-96 \times 10^{-9}$ & $\sim 15$ & $\sim 80$ & $(3)$ \\
\hline
\end{tabular}

Notes. $\chi(0)$ is the fractional abundance of each molecule measured towards the central driving protostar L1157-mm.

References. (1) Bachiller \& Pérez Gutiérrez (1997); (2) Vasta et al. (2012); (3) Rodríguez-Fernández et al. (2010).

Bachiller \& Pérez Gutiérrez 1997; Rodríguez-Fernández et al. 2010). Tafalla \& Bachiller (1995) specifically finds that SO and $\mathrm{SO}_{2}$ exhibit enhancement factors within L1157-B2 (relative to L1157-mm) of between 60-100 and 20, respectively. Meanwhile, they also find that the enhancement factors for L1157-B1 are 50-70 and 8 . HNCO is thought to form efficiently on grain surfaces, whilst $\mathrm{S}$-bearing species like $\mathrm{SO}$ and $\mathrm{SO}_{2}$ form in the gas-phase with sputtered $\mathrm{S}$ from the grains themselves (Allen \& Robinson 1977; Charnley 1997; Garrod et al. 2008). The older dynamical age of $\mathrm{B} 2$ relative to $\mathrm{B} 1$ could lend credence to the idea that B2 has simply had more time than B1 to chemically process the sputtered material, hence the more luminous species like HNCO and S-bearing species. Table 1 lists further molecules observed within L1157 and their enhancement factors, where $f_{\text {enhance }}=\chi(R) / \chi(0)$. Importantly these enhancement factors, as well as their associated abundances, are subject to large uncertainties arising from the assumption that the observed lines are both optically thin and thermalised.

To date studies such as those by Vasta et al. (2012) have not yet been able to determine with certainty the prevalent shock type within B2, though Vasta et al. (2012) does allude to the possibility of a J-type shock component within L1157-B2. GómezRuiz et al. (2016) use $\mathrm{NH}_{3}$ and $\mathrm{H}_{2} \mathrm{O}$ abundances, alongside model predictions, to trace shock temperature within L1157's lobes. Gómez-Ruiz et al. (2016) finds that whilst a proper line radiative transfer model is needed for proper computation, the best matching model for L1157-B2 is one with $n_{\mathrm{H}} \approx 10^{3} \mathrm{~cm}^{-3}$ and $v_{\mathrm{s}} \approx 10 \mathrm{~km} \mathrm{~s}^{-1}$.

\section{Shock modelling}

Our parameterised model is based on the MHD code mhd_vode (Flower \& Des ForÉts 2015). mhd_vode is an ideal-MHD, 1D, two-fluid simulation of both C-type and J-type shocks that computes chemistry in parallel with its physics. This model is built as a module to the time-dependent chemical code UCLCHEM (Holdship et al. 2017).

UCLCHEM is a diverse code, and its modularised functionality lends itself to a host of different astrochemical problems and environments. For a full description of UCLCHEM's operation see Holdship et al. (2017) as well as the documentation hosted online ${ }^{1}$. In brief, UCLCHEM is constructed so as to follow a two-phase computation. Firstly an ambient medium of user-supplied temperature, density and chemical composition undergoes an isothermal collapse as described by Rawlings et al. (1992) to a user-supplied final density. The chemical composition of a 1D parcel is therefore followed during collapse, and thus informs the chemical conditions for phase 2. During phase 2, the relevant physics supplied via a user module

\footnotetext{
1 https://uclchem.github.io/
}

is computed and used to inform the rates of reactions within the chemical network. Our J-type shock module is built so as to follow this methodology.

\subsection{J-type shock parameterisation}

To construct our parameterised model we first noted that, as described by Zel'dovich \& Raizer (1967), shocks can generally be discretised into four regions: the precursor, the shock-front, the post-shock relaxation layer and the thermalisation layer. We neglect the radiative precursor component of the shock in our models, as J-type shocks with $v_{\mathrm{s}}<80 \mathrm{~km} \mathrm{~s}^{-1}$ have been found to have negligible radiative precursor components, therefore playing no role in either the shock structure or the shock chemistry (Hollenbach \& McKee 1989; Flower et al. 2003b). We also neglect the thermalisation layer, instead focusing on the shock-front and the post-shock relaxation layer as sole sources of chemical evolution. We assume that the post-shock gas cools to its initial temperature in the post-shock relaxation layer.

To build the shock-front, we ran a grid of mhd_vode models with the magnetic field $B=0 \mathrm{G}$ and interstellar values for cosmic-ray ionisation rate $\zeta_{\mathrm{CR}}$ and radiation field, so as to quantify the trend in temperature and density, as well as the shock-front duration $t_{\text {front }}$, across the parameter space we were exploring. $t_{\text {front }}$, in units of $\mathrm{s}$, is described by Eq. (1).

$t_{\text {front }}=\frac{\left(\sqrt{2} \pi\left(5.76 \times 10^{-16}\right)^{-1}\right)}{v_{\mathrm{s}} \times 10^{6}}$

where $v_{\mathrm{s}}$ represents the initial shock velocity in $\mathrm{km} \mathrm{s}^{-1}$. The increase in temperature and density within the shock-front was found to be best described by $T=T_{\max }\left(t / t_{\text {front }}\right)^{2}$ in $\mathrm{K}$ and $n_{\mathrm{H}}=4 n_{\mathrm{H}_{\text {initial }}}\left(t / t_{\text {front }}\right)^{4}$ in $\mathrm{cm}^{-3}$. For $t<t_{\text {front }}$ we assume that the Rankine-Hugoniot conditions (Rankine 1870; Hugoniot 1889) hold such that the density $n_{\mathrm{H}}$ increases to $\approx 4$ times its initial value whilst the temperature $T$ increases to its maximum obtainable value, $T_{\max } . T_{\max }$ is determined by $T_{\max }=5 \times 10^{3}\left(v_{\mathrm{s}} / 10\right)^{2}$ in K (Williams \& Viti 2013).

After the shock-front, the shocked gas begins to cool, representing the post-shock relaxation layer where $t>t_{\text {front }}$ and $t<t_{\text {shock }} t_{\text {shock }}$ was obtained by fitting a polynomial to a range of shock timescales from mhd_vode models and is described by Eq. (2).

$t_{\text {shock }}=\frac{t_{\text {year }} \times 10^{6}}{n_{\mathrm{H}_{\text {initial }}}}$

where $t_{\text {year }}$ is the number of seconds in 1 year and $n_{\mathrm{H}_{\text {initial }}}$ is the initial pre-shock number density in $\mathrm{cm}^{-3}$. The factor of $10^{6}$ acts as a normalising density such that $t_{\text {shock }}$ has units of $\mathrm{s}$. 
Within this layer, the temperature and density equations take the forms described in Eqs. (3) and (4).

$T=T_{\max } e^{-\lambda_{T}\left(\frac{t}{t_{\text {shock }}}\right)}$

$n=4 n_{\text {initial }} e^{\lambda_{n}\left(\frac{t}{t_{\text {shock }}}\right)}$.

Equation (3) has units of K, whilst Eq. (4) has units of $\mathrm{cm}^{-3}$. This therefore allows the gas to cool following a decaying exponential law, whilst the gas also increases in density to $n_{\mathrm{H}_{\max }}$, which is itself derived from mhd_vode grids. $n_{\mathrm{H}_{\max }}$ is defined as $n_{\mathrm{H}_{\max }}=\left(v_{\mathrm{s}} \times n_{\mathrm{H}_{\text {initial }}}\right) \times 10^{2}$ in units of $\mathrm{cm}^{-3}$. The constants $\lambda_{T}$ and $\lambda_{n}$ in Eqs. (3) and (4) are described by $\lambda_{T}=\ln \left(\frac{T_{\max }}{T_{\text {initial }}}\right)$ and $\lambda_{n}=\ln \left(\frac{n_{\max }}{n_{\text {initial }}}\right)$. At $t>t_{\text {shock }}$, we assume that the gas has cooled back to its initial temperature $T_{\text {initial }}$. We assume a steady-state profile for both $T$ and $n$, and discuss the validity of this approximation in Sect. 4.1 .

\subsection{C-type shock parameterisation}

UCLCHEM implements a version of the parameterised C-type shock from Jiménez-Serra et al. (2008). The UCLCHEM implementation is described in more detail, as well as demonstrated to good effect, in Holdship et al. (2017).

Similarly to the J-type shock parameterisation presented in Sect. 3, Jiménez-Serra et al. (2008) approximates the physical shock structure using analytical equations for $T$ and $n_{\mathrm{H}}$ alongside the velocity of the ions and neutrals, $v_{\mathrm{i}}$ and $v_{\mathrm{n}}$ respectively (see Appendix A of Jiménez-Serra et al. 2008 for further details). They also make use of results from Draine et al. (1983) to parameterise the maximum shock temperature $T_{\max }$ as a function of shock velocity $v_{\mathrm{s}}$. It is this temperature that is shown for the C-type shock in Table 2.

Jiménez-Serra et al. (2008) also present, in Appendix B, a fractional sputtering treatment of grain mantle species such $\mathrm{Si}$, $\mathrm{CH}_{3} \mathrm{OH}$, and $\mathrm{H}_{2} \mathrm{O}$. UCLCHEM now supports this sputtering implementation. In summary, rather than an instantaneous ejection of the mantle into the gas phase when the saturation time $t_{\mathrm{sat}}{ }^{2}$ is exceeded, only a fraction of the species abundance will be released from the mantles and/or ices at any given timestep providing the drift velocity between the neutrals and ions, as well as the impact energy, is sufficient to sputter material.

Of critical importance in C-type shock formation is the magnetic field, $B$. UCLCHEM's C-type shock implementation assumes the $B$-field (in $\mu \mathrm{G}$ ) scales according to the emperical law defined in Draine et al. (1983), i.e. $B_{0}=b_{0} \sqrt{n_{\mathrm{H}}}$ where $b_{0}$ is the magnetic scaling parameter and $n_{\mathrm{H}}$ the Hydrogen number density. Much like Draine et al. (1983), we fix $b_{0}$ as 1 , thus allowing the magnetic field to scale with $\sqrt{n_{\mathrm{H}}}$ as defined in Table 4 of Draine et al. (1983). According to this relation, at $n_{\mathrm{H}}=10^{3} \mathrm{~cm}^{-3}$ the magnetic field has a field strength of $B_{0}=10 \mu \mathrm{G}$ whilst at $n_{\mathrm{H}}=10^{6} \mathrm{~cm}^{-3}$ the magnetic field has field strength $B_{0}=1 \mathrm{mG}$, both of which are consistent with Table 4 of Draine et al. (1983).

\subsection{Computational grid}

Gómez-Ruiz et al. (2016) finds the best fit profile to $\mathrm{NH}_{3}$ and $\mathrm{H}_{2} \mathrm{O}$ abundances in L1157-B2 is one with $v_{\mathrm{s}}=10 \mathrm{~km} \mathrm{~s}^{-1}$ and $n_{\mathrm{H}}=10^{3} \mathrm{~cm}^{-3}$, and we use this as to inform our choice of initial conditions for our grid of models.

\footnotetext{
$2 t_{\mathrm{sat}}$ is defined as the time for which the logarithmic difference of the $\mathrm{Si}$ abundance between two consecutive timesteps $t_{i+1}$ and $t_{i}$ is $\left|\log _{10} \chi\left(m_{i+1}\right)-\log _{10} \chi\left(m_{i}\right)\right|<0.1$.
}

Table 2. Grid of models used to compute simulations.

\begin{tabular}{|c|c|c|c|c|}
\hline \multirow[t]{2}{*}{ Model } & \multirow[t]{2}{*}{$n_{\mathrm{H}}\left[\mathrm{cm}^{-3}\right]$} & \multirow[t]{2}{*}{$v_{\mathrm{s}}\left[\mathrm{km} \mathrm{s}^{-1}\right]$} & \multicolumn{2}{|c|}{$T_{\max }[\mathrm{K}]$} \\
\hline & & & C-type & J-type \\
\hline 1 & $10^{3}$ & 5 & 85 & 1250 \\
\hline 2 & $10^{4}$ & 5 & 85 & 1250 \\
\hline 3 & $10^{5}$ & 5 & 85 & 1250 \\
\hline 4 & $10^{6}$ & 5 & 85 & 1250 \\
\hline 5 & $10^{3}$ & 6 & 131 & 1800 \\
\hline 6 & $10^{4}$ & 6 & 131 & 1800 \\
\hline 7 & $10^{5}$ & 6 & 131 & 1800 \\
\hline 8 & $10^{6}$ & 6 & 131 & 1800 \\
\hline 9 & $10^{3}$ & 7 & 178 & 2450 \\
\hline 10 & $10^{4}$ & 7 & 178 & 2450 \\
\hline 11 & $10^{5}$ & 7 & 178 & 2450 \\
\hline 12 & $10^{6}$ & 7 & 178 & 2450 \\
\hline 13 & $10^{3}$ & 8 & 225 & 3200 \\
\hline 14 & $10^{4}$ & 8 & 225 & 3200 \\
\hline 15 & $10^{5}$ & 8 & 225 & 3200 \\
\hline 16 & $10^{6}$ & 8 & 225 & 3200 \\
\hline 17 & $10^{3}$ & 9 & 273 & 4050 \\
\hline 18 & $10^{4}$ & 9 & 273 & 4050 \\
\hline 19 & $10^{5}$ & 9 & 273 & 4050 \\
\hline 20 & $10^{6}$ & 9 & 273 & 4050 \\
\hline 21 & $10^{3}$ & 10 & 323 & 5000 \\
\hline 22 & $10^{4}$ & 10 & 323 & 5000 \\
\hline 23 & $10^{5}$ & 10 & 323 & 5000 \\
\hline 24 & $10^{6}$ & 10 & 323 & 5000 \\
\hline 25 & $10^{3}$ & 11 & 373 & 6050 \\
\hline 26 & $10^{4}$ & 11 & 373 & 6050 \\
\hline 27 & $10^{5}$ & 11 & 373 & 6050 \\
\hline 28 & $10^{6}$ & 11 & 373 & 6050 \\
\hline 29 & $10^{3}$ & 12 & 424 & 7200 \\
\hline 30 & $10^{4}$ & 12 & 424 & 7200 \\
\hline 31 & $10^{5}$ & 12 & 424 & 7200 \\
\hline 32 & $10^{6}$ & 12 & 424 & 7200 \\
\hline 33 & $10^{3}$ & 13 & 477 & 8450 \\
\hline 34 & $10^{4}$ & 13 & 477 & 8450 \\
\hline 35 & $10^{5}$ & 13 & 477 & 8450 \\
\hline 36 & $10^{6}$ & 13 & 477 & 8450 \\
\hline 37 & $10^{3}$ & 14 & 530 & 9800 \\
\hline 38 & $10^{4}$ & 14 & 530 & 9800 \\
\hline 39 & $10^{5}$ & 14 & 530 & 9800 \\
\hline 40 & $10^{6}$ & 14 & 530 & 9800 \\
\hline 41 & $10^{3}$ & 15 & 585 & 11250 \\
\hline 42 & $10^{4}$ & 15 & 585 & 11250 \\
\hline 43 & $10^{5}$ & 15 & 585 & 11250 \\
\hline 44 & $10^{6}$ & 15 & 585 & 11250 \\
\hline
\end{tabular}

Notes. The velocity $v_{\mathrm{s}}$, density $n_{\mathrm{H}}$ and maximum temperature achieved in both C-type and J-type shocks, $T_{\max }$, is shown. Each model is run twice: once for a C-type shock and once for a J-type shock.

Table 2 shows the range of parameters used to compute this grid. For a J-type shock $T_{\max }$ is determined as discussed, whilst for a C-type shock $T_{\max }$ is determined according to the parameterisation discussed in Jiménez-Serra et al. (2008) (see Sect. 3.2).

We also account for the initial C-type shock conditions published by other authors so as to verify the feasibility of C-type 


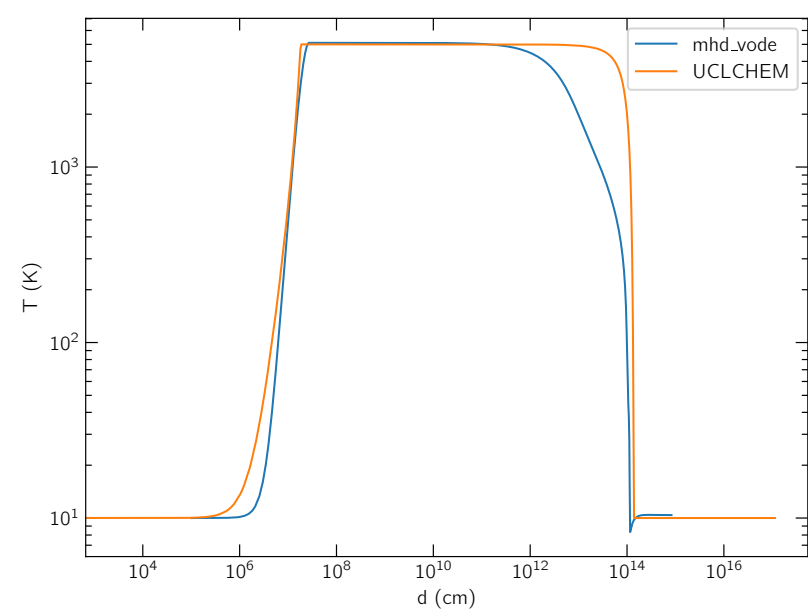

Fig. 2. Comparing the temperature structure of a J-type shock with $v=$ $10 \mathrm{~km} \mathrm{~s}^{-1}$ and $n_{\mathrm{H}}=10^{3} \mathrm{~cm}^{-3}$ computed with the model presented in this paper and the mhd_vode model by Flower \& Des ForÉts (2015). Good agreement is observed, despite our approximation not recovering all of the features in the mhd_vode profile. The model built for UCLCHEM is also isothermal such that it cools back to its initial temperature, whereas mhd_vode is not despite it cooling to $\approx 10 \mathrm{~K}$ in this instance.

shock formation at the conditions considered. For example Holdship et al. (2017) identifies C-type shock-tracing molecules for a range of different physical shock conditions to a lower limit of $v_{\mathrm{s}}=10 \mathrm{~km} \mathrm{~s}^{-1}$ and $n_{\mathrm{H}}=10^{3} \mathrm{~cm}^{-3}$. Furthermore, Draine et al. (1983) identify the maximum shock temperature for a range of different C-type shocks with a lower limit of $v_{\mathrm{s}}=5 \mathrm{~km} \mathrm{~s}^{-1}$ and $n_{\mathrm{H}}=10^{2} \mathrm{~cm}^{-3}$ with a $B$ field defined by $B=10 \mu \mathrm{G}$. Finally, Godard et al. (2019) investigate the formation of a range of different shock types under different $B$ fields and irradiated conditions. They highlight $\mathrm{C}$-type shocks forming between $v_{\mathrm{s}}=$ $5-20 \mathrm{~km} \mathrm{~s}^{-1}$ and $n_{\mathrm{H}}=10^{2}-10^{5} \mathrm{~cm}^{-3}$ under a range of $B$ fields from $B=1 \mu \mathrm{G}$ to $B=3 \mathrm{mG}$. Our parameters fit comfortably into this published range and we therefore assume that $C$-type shock formation at these conditions is entirely feasible.

For each $v_{\mathrm{s}}$ and $n_{\mathrm{H}}$ within Table 2, the fractional abundance of 215 individual molecules, including $\mathrm{H}_{2} \mathrm{O}, \mathrm{HCN}, \mathrm{CH}_{3} \mathrm{OH}$, SO and $\mathrm{SO}_{2}$, was computed for both $\mathrm{C}$-type and J-type shocks. This was achieved by coupling the physical shock computations from within the physics modules of UCLCHEM to a chemical network of 2456 reactions. Further details of the network are discussed later in this section. We plot the fractional abundance of a molecule against distance through the shock, up to the C-type shock dissipation length as determined by Jiménez-Serra et al. (2008). The dissipation length is defined as the distance over which the velocity of the ions and neutrals equalises (Draine 1980). As a J-type shock consists of one fluid that encompasses both ions and neutrals, the concept of a dissipation length does not apply. Instead, we plot the J-type shock fractional abundance up to the cooling length of the shock, beyond which the gas has reached equilibrium. As the fluids within a C-type shock also reach equilibrium at the dissipation length, we assume the two distance scales are comparable.

Using these plots, the abundance trends were then compared between shock types to better understand the behaviour of species under different shock conditions. Of particular interest in this study was the enhancement factors observed in Table 1, as this forms the signature of shock passage and therefore the best diagnostic of shock type in a shocked region.

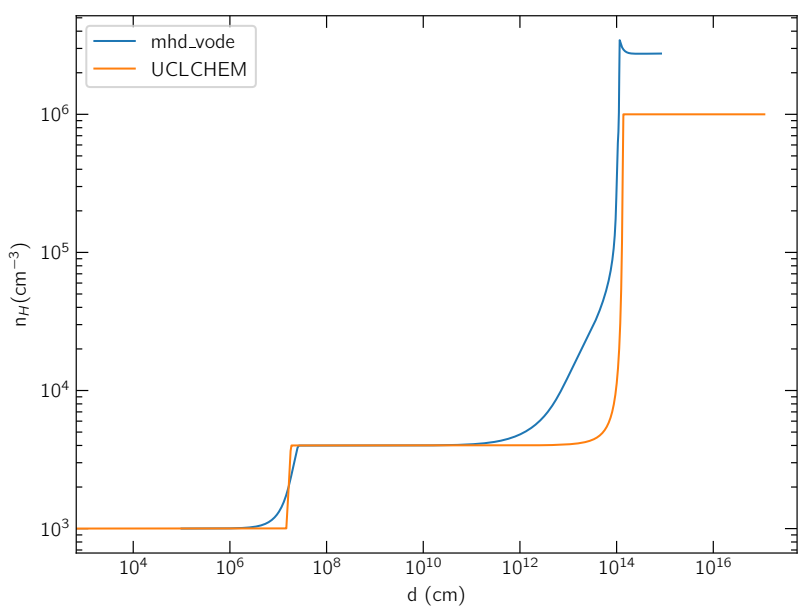

Fig. 3. Like Fig. 2, here we compare the density profiles for the J-type shock in mhd_vode, as well as the model presented in this paper. Good agreement is again observed, despite the lack of inflexion point recovery.

Principal to this enhancement factor analysis is the assumption that the pre-shock gas is homogeneous throughout L1157 and the surrounding region, therefore allowing the fractional abundance at $t \approx 0$ years in phase 2 to be consistent with nonshocked regions of gas outside the shocked knots. This may only be true for the B2 region, as previous work (Viti et al. 2011) has indicated that a pre-existing, non-homogeneous clump is required for the extant chemistry at $\mathrm{B} 1$ to occur. To date, there is no such evidence observed towards B2, hence the homogeneous pre-shock gas assumption. Using this, we can also compute enhancement factors relative to the fractional abundance at $t \approx 0$ years, thus allowing direct comparison to the abundances and enhancement factors listed in Table 1.

The chemical network used to compute the abundances considered is based on the network described by Holdship et al. (2017). To summarise in brief, we use a reduced form of the UMIST database (McElroy et al. 2013) to build a network of gasphase reactions. We also include a dust-grain reaction network that allows for freeze out with hydrogenation and both thermal and non-thermal desorption.

\section{Results}

\subsection{Model comparison}

Figure 2 shows the profile of temperature $T$, whilst Fig. 3 shows the profile of density $n_{\mathrm{H}}$ for both mhd_vode and the model presented in this work.

Qualitatively comparing the $T$ profiles in Fig. 2 we observe good agreement between the mhd_vode model and the UCLCHEM model's computation of $T$ in the shock-front described by Eq. (3). Both models reach approximately the same $T_{\max }$ over an almost identical distance despite the UCLCHEM model beginning its heating prior to the mhd_vode model.

Further agreement is observed until $d \approx 10^{11} \mathrm{~cm}$, whereby mhd_vode begins to cool rapidly, further exhibiting an inflexion point at $d \approx 10^{13} \mathrm{~cm}$, causing $T$ to drop from $5000 \mathrm{~K}$ to $300 \mathrm{~K}$. As a result agreement diverges between $10^{11}<d<10^{14} \mathrm{~cm}$. This departure is a consequence of mhd_vode's radiative cooling, which UCLCHEM does not implement.

Furthermore mhd_vode does not explicitly cool back to its initial temperature, though it does reach an equilibrium 


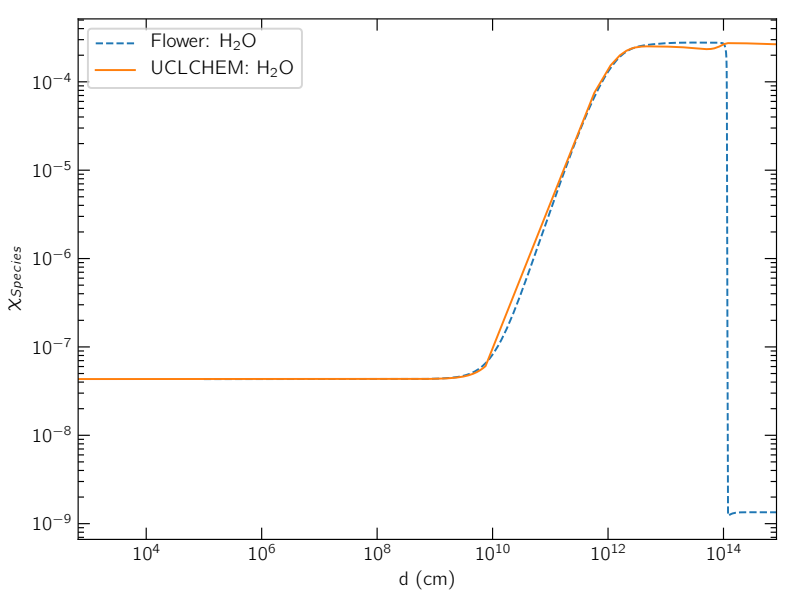

Fig. 4. Evolution of $\mathrm{H}_{2} \mathrm{O}$ during the shock referenced in Figs. 2 and 3 in both mhd_vode and UCLCHEM. Within this figure, sputtering has been deactivated in UCLCHEM for the purposes of comparison. This implies that only gas-phase chemical reactions are active in these simulations so that the effect of the differences in the temperature profiles between mhd_vode and our approximation can be fairly evaluated. The abundance evolution of $\mathrm{H}_{2} \mathrm{O}$ up to $d \approx 10^{13} \mathrm{~cm}$ is in almost perfect agreement. This is in spite of the lack of inflexion point in both $T$ and $n$ between $10^{11}<d<10^{14} \mathrm{~cm}$. This proves that such a departure has negligible effect during the shock. mhd_vode manually cools $\mathrm{H}_{2} \mathrm{O}$, hence the decrease in abundance at $d \approx 10^{\overline{14}} \mathrm{~cm}$. UCLCHEM does not implement this cooling.

temperature very close to that of its initial temperature. Figure 2 shows the mhd_vode model cooling its gas to $\approx 10 \mathrm{~K}$ after $d \approx 10^{14} \mathrm{~cm}$. The parameterised model presented here explicitly assumes that the gas cools back to $T_{\text {initial }}$. In Fig. 2 this is $10 \mathrm{~K}$.

Comparisons between $n_{\mathrm{H}}$ models in Fig. 3 show qualitatively less agreement, especially regarding the peak $n_{\mathrm{H}}$. However, the UCLCHEM peak $n_{\mathrm{H}}$ is within a factor of 2 of the mhd_vode model.

The inflexion point highlighted in Fig. 2 is also present within Fig. 3 at the same time. Similarly to before, we do not attempt to recover this feature. To assess the effect that this missing feature has on our approximation, and the subsequent chemistry that this model is used to inform, we directly compare the chemistry of $\mathrm{H}_{2} \mathrm{O}$ between mhd_vode and UCLCHEM. This is seen in Fig. 4. Importantly, the public version of mhd_vode used in this study does not include sputtering. Therefore for this comparison, we disable UCLCHEM's sputtering treatment to compare chemistry with the same major gas-grain treatments present.

For the same initial conditions, mhd_vode and UCLCHEM produce the same $\mathrm{H}_{2} \mathrm{O}$ abundance behaviour despite UCLCHEM not recovering the observed inflexion point. This is true up to $d=10^{14} \mathrm{~cm}$, where mhd_vode radiatively cools $\mathrm{H}_{2} \mathrm{O}$, causing its abundance to drop sharply. UCLCHEM does not implement this form of cooling and so the $\mathrm{H}_{2} \mathrm{O}$ abundance does not drop sharply until a much greater distance into the shock.

Given that our model is never more than a factor of 3 away from the mhd_vode equivalent, and that the shocked $\mathrm{H}_{2} \mathrm{O}$ abundances are in almost perfect agreement, we consider our parameterisation of a J-type shock a good approximation of an equivalent shock model from an ideal-MHD simulation such as mhd_vode.

Part of our model is the simplifying assumption that the shock is steady-state. This is valid and physically justified as long as the cooling time of the shock is shorter than the time for which the shock velocity and the pre-shock conditions of the gas can change (Martinez 2009). In our grid runs, we switch back on grain chemistry and assume that the mantle ices instantaneously evaporate if the temperature of the gas $T>100 \mathrm{~K}$. This is derived from plots within Fraser et al. (2001). We also assume that any species that have formed in the solid-state on the dust-grain will co-desorb alongside the mantle ices.

We note that the instantaneous evaporation of the ices in J-type shocks occurs before sputtering takes place. This is fully justified since this is the expected behaviour from the J-type shock's rapid heating of gas and dust at the sharp shock front. For C-type shocks, we consider both processes, ice evaporation when $T$ exceeds $100 \mathrm{~K}$ and sputtering. Since $T$ is significantly lower in C-type shocks, evaporation is less efficient and so sputtering is more effective at releasing a fractional amount of the ices into the gas phase (see Jiménez-Serra et al. 2008, for details on the fractional sputtering technique implemented in UCLCHEM).

The qualitative agreement noted thus far between mhd_vode model and our parameterised model validates our steady-state assumption for the initial shock conditions applied here.

\subsection{Identifying J-type shock behaviour}

To identify unique J-type shock behaviour, we determine the average abundance across the post-shock region ${ }^{3}$ arising as a result of both J-type and C-type shocks for each model within our grid, and express the ratio of these two average abundances, $\chi(J) / \chi(C)$. J-type shock enhanced molecules are therefore molecules that have $\chi(J) / \chi(C) \gg 1$.

To assess the distribution of ratios across the entire grid we bin each model by its values of $v_{\mathrm{s}}$ and $n_{\mathrm{H}}$ and construct a $2 \mathrm{D}$ colour plot. The colour within each bin represents the ratio of the average post-shock abundances, $\chi(J) / \chi(C)$, up to the dissipation length (or equivalent) for both shock types.

We also use the enhancement factor, $f_{\text {enhance}}$, as a diagnostic. We define $f_{\text {enhance }}$ in Eq. (5).

$f_{\text {enhance }}=\frac{\chi(R)}{\chi(0)}$

$\chi(R)$ is the fractional abundance of the shocked molecule, whilst $\chi(0)$ is the fractional abundance of the molecule in a quiescent state. Within this study, we take $\chi(0)$ to be the abundance at simulation time $t \approx 0$ years before any sputtering takes place. $f_{\text {enhance }}$ is therefore directly comparable to $f$ in Table 1 .

This analysis was performed for a range of different known shock-tracing molecules including $\mathrm{CH}_{3} \mathrm{OH}, \mathrm{H}_{2} \mathrm{O}, \mathrm{SO}, \mathrm{SO}_{2}$ and $\mathrm{HCN}$. We also investigated the behaviour of molecules such as $\mathrm{SiO}$, however our analysis indicated that its behaviour was not noteworthy at the considered conditions. We attribute this to our shock velocities $v_{\mathrm{s}}$ being too slow to efficiently sputter and form $\mathrm{SiO}$.

\subsection{1. $\mathrm{CH}_{3} \mathrm{OH}$}

Figure 5 shows the ratio of the average post-shock abundances up to the dissipation length (or equivalent) for each shock type. It is computed for C-type shock and J-type shock enhanced

3 For the J-type shocks we define the post-shock region as that found between the shock-front and the end of the cooling region; while for a C-type shock, the post-shock region coincides with the length of the dissipation region of the shock. 


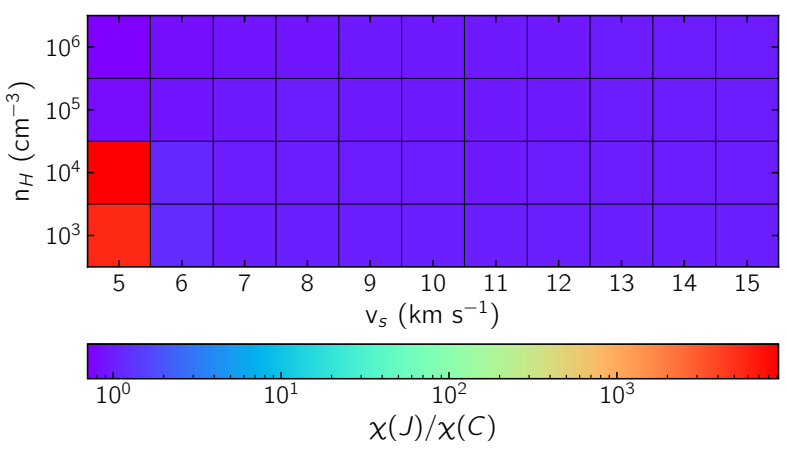

Fig. 5. Ratio of the average J-type enhanced $\mathrm{CH}_{3} \mathrm{OH}$ abundance to the average C-type enhanced $\mathrm{CH}_{3} \mathrm{OH}$ abundance. As is clear, there is no chemical difference between J-type and C-type enhanced $\mathrm{CH}_{3} \mathrm{OH}$, except at low $v_{\mathrm{s}}$ and low $n_{\mathrm{H}}$. This major difference - a factor of $8000-$ arises as a result of the C-type shock failing to sputter grain surface material whilst the J-type shock instantaneously evaporates grain surface $\mathrm{CH}_{3} \mathrm{OH}$.

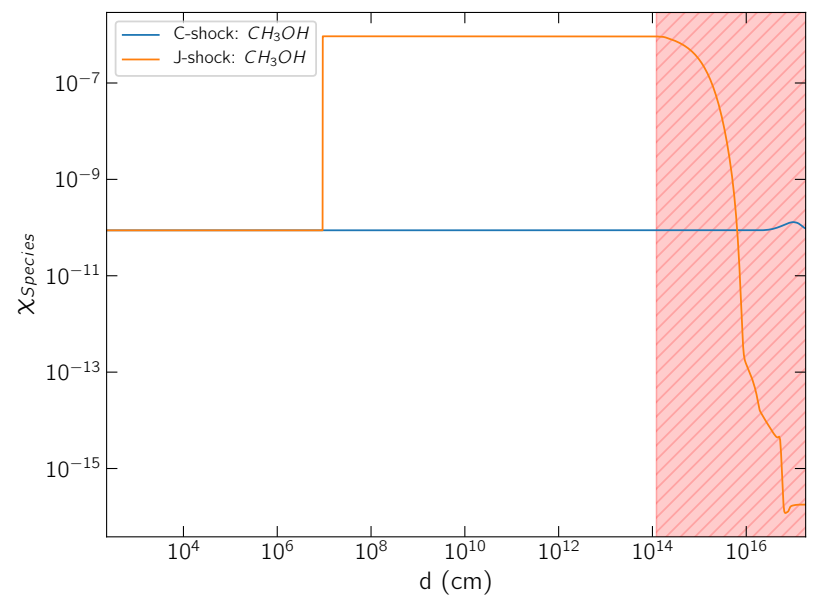

Fig. 6. $\mathrm{CH}_{3} \mathrm{OH}$ abundances for a shock with initial velocity $v_{\mathrm{s}}=$ $5 \mathrm{~km} \mathrm{~s}^{-1}$ and density $n_{\mathrm{H}}=10^{3} \mathrm{~cm}^{-3}$. The shaded red region indicates the region beyond which the J-type shock has cooled to its equilibrium temperature.

$\mathrm{CH}_{3} \mathrm{OH}$ for each model in the grid described in Table 2. Within this figure, $\chi(C)$ represents the average gas-phase abundance in a C-type shock achieved up to the dissipation length, whilst $\chi(J)$ is the average gas-phase abundance up to the cooling length for a J-type shock.

Figure 5 shows that there is essentially no difference in chemistry between shock type for $\mathrm{CH}_{3} \mathrm{OH}$, except the models where $v_{\mathrm{s}}=5 \mathrm{~km} \mathrm{~s}^{-1}$ and $n_{\mathrm{H}}=10^{3} \mathrm{~cm}^{-3}$ as well as $n_{\mathrm{H}}=$ $10^{4} \mathrm{~cm}^{-3}$.

This unique disparity stems from the stark difference in gasgrain behaviour between shock types under these conditions. As Fig. 6 shows, the $\mathrm{CH}_{3} \mathrm{OH}$ abundance sharply increases as a result of instantaneous evaporation at $d \approx 10^{7} \mathrm{~cm}$ in the J-type shock. In the C-type shock, neither evaporation nor sputtering occurs, meaning the $\mathrm{CH}_{3} \mathrm{OH}$ abundance remains relatively consistent throughout the shock.

This is confirmed in Figs. 6 and 7, which shows the $\mathrm{CH}_{3} \mathrm{OH}$ abundance as a function of distance through both C-type and J-type shocks with velocity $v_{\mathrm{s}}=5 \mathrm{~km} \mathrm{~s}^{-1}$ and density $n_{\mathrm{H}}=$ $10^{3} \mathrm{~cm}^{-3}$ and $n_{\mathrm{H}}=10^{6} \mathrm{~cm}^{-3}$.

At conditions excluding those already discussed, sputtering becomes efficient, hence the abundance ratios in Fig. 5 tending to



Fig. 7. $\mathrm{CH}_{3} \mathrm{OH}$ abundances for a shock with initial velocity $v_{\mathrm{s}}=$ $5 \mathrm{~km} \mathrm{~s}^{-1}$ and density $n_{\mathrm{H}}=10^{6} \mathrm{~cm}^{-3}$. Again, the shaded red region indicates the region beyond which the J-type shock has cooled to its equilibrium temperature.

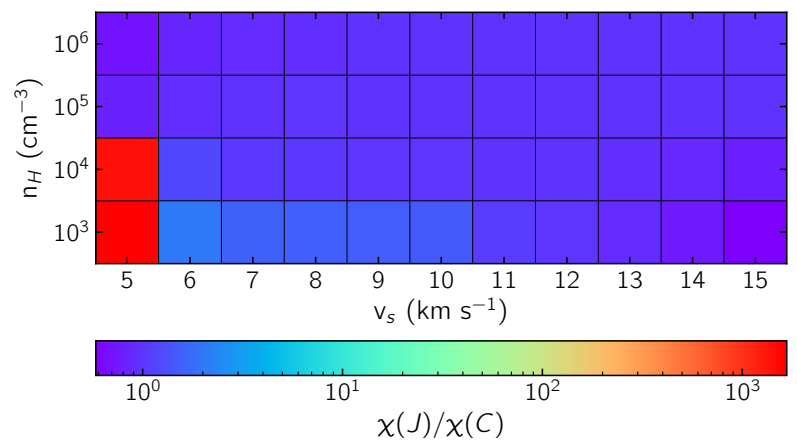

Fig. 8. Ratio of the average J-type enhanced $\mathrm{H}_{2} \mathrm{O}$ abundance to the average $\mathrm{C}$-type enhanced $\mathrm{H}_{2} \mathrm{O}$ abundance. The largest difference between average shock type abundance is at $v_{\mathrm{s}}=5 \mathrm{~km} \mathrm{~s}^{-1}$ and $n_{\mathrm{H}}=10^{3} \mathrm{~cm}^{-3}$ and $n_{\mathrm{H}}=10^{4} \mathrm{~cm}^{-3}$ where the ratio exceeds 1000 .

1 uniformly throughout the rest of the grid as a result of $\mathrm{CH}_{3} \mathrm{OH}$ being co-desorbed in a J-type shock and sputtered in a C-type shock in equal measure. Importantly, following injection/sputtering there is minimal subsequent gas-phase chemistry in either shock, hence reinforcing the common abundances achieved in Fig. 5 regardless of shock type.

As a result of the J-type shock's rapid heating, instantaneous evaporation occurs well before any sputtering activity in a C-type shock. In both shocks, the same amount of $\mathrm{CH}_{3} \mathrm{OH}$ is released from the dust-grains owing to self-consistent initial conditions from phase 1 of UCLCHEM.

\subsection{2. $\mathrm{H}_{2} \mathrm{O}$}

The abundance ratios for $\mathrm{H}_{2} \mathrm{O}$ is shown in Fig. 8. Much like $\mathrm{CH}_{3} \mathrm{OH}$ in Sect. 4.2.1, $\mathrm{H}_{2} \mathrm{O}$ behaves similarly at $v_{\mathrm{s}}=5 \mathrm{~km} \mathrm{~s}^{-1}$ and $n_{\mathrm{H}}=10^{3} \mathrm{~cm}^{-3}$ as well as $n_{\mathrm{H}}=10^{4} \mathrm{~cm}^{-3}$ owing to the same processes; in other words the J-type shock instantaneously evaporates material whilst the C-type shock neither sputters nor evaporates.

Outside of this, the biggest difference between C-type and J-type shocks peaks at $v_{\mathrm{s}}<10 \mathrm{~km} \mathrm{~s}^{-1}$ and $n_{\mathrm{H}}=10^{3} \mathrm{~cm}^{-3}$. The enhancement factors drop off to $\approx 1$ at velocities and densities greater than these. 


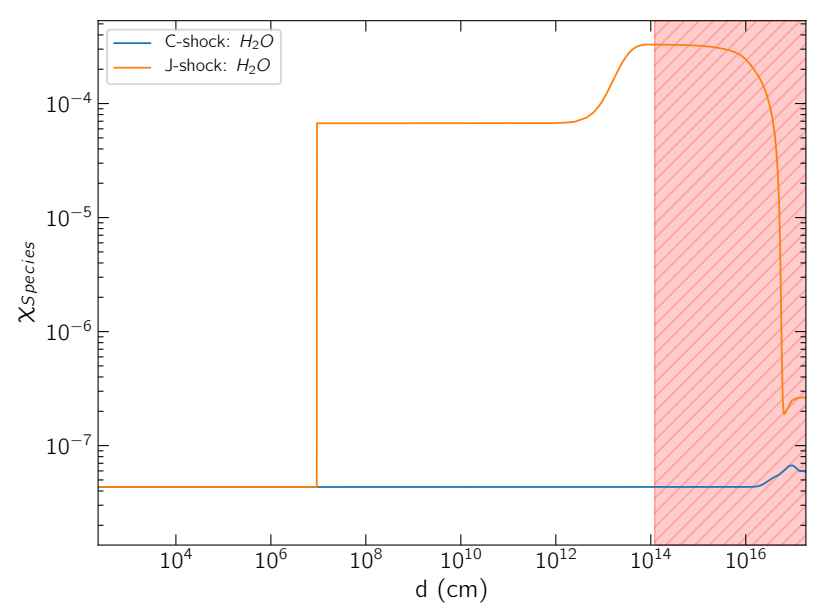

Fig. 9. $\mathrm{H}_{2} \mathrm{O}$ abundances for a shock with initial velocity $v_{\mathrm{s}}=5 \mathrm{~km} \mathrm{~s}^{-1}$ and density $n_{\mathrm{H}}=10^{3} \mathrm{~cm}^{-3}$.

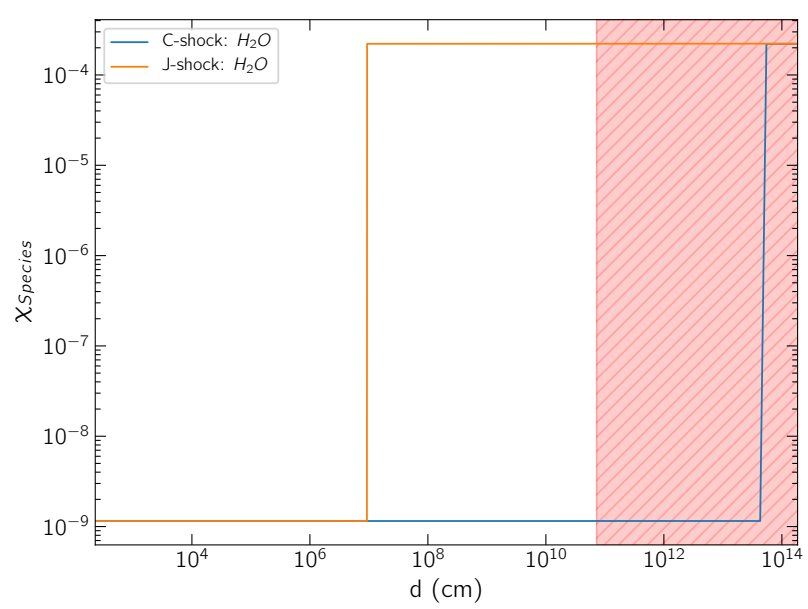

Fig. 10. $\mathrm{H}_{2} \mathrm{O}$ abundances for a shock with initial velocity $v_{\mathrm{s}}=5 \mathrm{~km} \mathrm{~s}^{-1}$ and density $n_{\mathrm{H}}=10^{6} \mathrm{~cm}^{-3}$.

Figures 9 and 10 shows the $\mathrm{H}_{2} \mathrm{O}$ abundances as a function of distance through the shock for C-type and J-type shocks with velocity $v_{\mathrm{s}}=5 \mathrm{~km} \mathrm{~s}^{-1}$ and density $n_{\mathrm{H}}=10^{3}$ and $n_{\mathrm{H}}=10^{6} \mathrm{~cm}^{-3}$.

In the J-type shock profiles from Figs. 9 and 10, the gas phase abundance of $\mathrm{H}_{2} \mathrm{O}$ increases sharply at $\approx 10^{7} \mathrm{~cm}$. This feature arises as a result of evaporation of the solid state material frozen on to the dust grains, e.g. the ices. The C-type shock may also undergo an increase in gas phase $\mathrm{H}_{2} \mathrm{O}$ at a later time in the shock as a result of sputtering, providing that the initial shock conditions enable the sputtering process. In our models, sputtering does not occur at $v_{\mathrm{s}}=5 \mathrm{~km} \mathrm{~s}^{-1}$ and $n_{\mathrm{H}}=10^{3} \mathrm{~cm}^{-3}$ as well as $n_{\mathrm{H}}=10^{4} \mathrm{~cm}^{-3}$, hence the large difference in average abundance at these models in Fig. 8.

Post-evaporation features within Figs. 9 and 10 begin to explain the more minor gas-phase enhancement in Fig. 8. For the J-type shock in Fig. 9, the abundance of $\mathrm{H}_{2} \mathrm{O}$ increases to a maximum of $\approx 3 \times 10^{-4}$, approximately 6 times the postevaporation abundance, at around $d \approx 10^{13} \mathrm{~cm}$. This effect is largest at $n_{\mathrm{H}}=10^{3} \mathrm{~cm}^{-3}$ and is present as $n_{\mathrm{H}}$ increases, though the magnitude of the gas-phase enhancement does decrease as $n_{\mathrm{H}}$ increases. At $n_{\mathrm{H}}=10^{6} \mathrm{~cm}^{-3}$ (Fig. 10) there is no postevaporation gas phase abundance change in $\mathrm{H}_{2} \mathrm{O}$, thus eliminating the effect altogether.

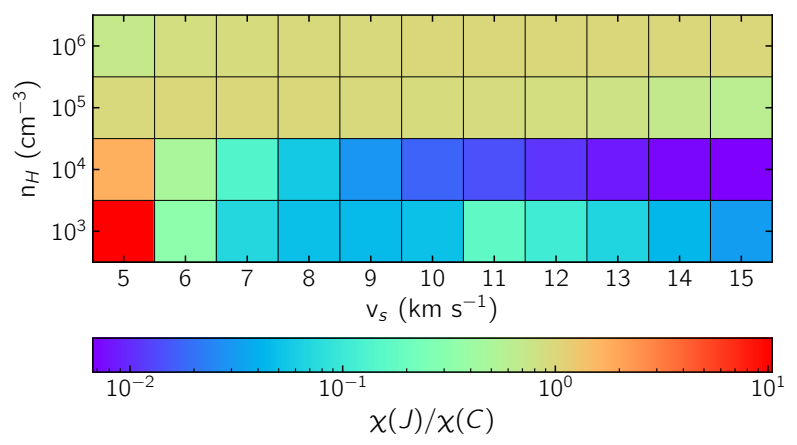

Fig. 11. Ratio of the average J-type enhanced SO abundance to the average C-type enhanced SO abundance. The largest difference between peak shock type abundance is at $n_{\mathrm{H}}=10^{3} \mathrm{~cm}^{-3}$. The shock conditions that produce unique chemistry in this parameter space are those with $n_{\mathrm{H}}<10^{5} \mathrm{~cm}^{-3}$.

Investigating the C-type shock in Figs. 9 and 10, we observe no post-sputtering increase in $\mathrm{H}_{2} \mathrm{O}$, regardless of $n_{\mathrm{H}}$. This, coupled with the decreasing gas-phase enhancement in the J-type as $n_{\mathrm{H}}$ increases, results in both shock types tending to the same abundance.

This explains why the largest enhancement is seen at low $v_{\mathrm{s}}$, low $n_{\mathrm{H}}$. As $n_{\mathrm{H}}$ increases, an overall decrease in the post-injection gas phase abundance change is observed, despite the evaporated $\mathrm{H}_{2} \mathrm{O}$ increasing with $n_{\mathrm{H}}$. As $v_{\mathrm{s}}$ increases, the peak temperature of the shock also rises, allowing gas-phase $\mathrm{H}_{2} \mathrm{O}$ to be destroyed. For a J-type shock, $\mathrm{H}_{2} \mathrm{O}$ destruction begins at $v_{\mathrm{s}}=11 \mathrm{~km} \mathrm{~s}^{-1}$ when $T_{\max }>6000 \mathrm{~K}$.

\subsubsection{SO}

Figure 11 shows the average abundance ratios for SO. Interestingly, Fig. 11 shows that SO is not produced more efficiently in a J-type shock than a C-type shock in our parameter space. In actuality, for $n_{\mathrm{H}}>10^{4} \mathrm{~cm}^{-3}$ the ratio $\chi(J) / \chi(C) \approx 1$, indicating that at high density both shocks are able to enhance SO to similar degrees.

The behaviour of SO at $n_{\mathrm{H}}<10^{4} \mathrm{~cm}^{-3}$ is starkly different. Considering the $n=10^{3} \mathrm{~cm}^{-3}$ row within Fig. 11 , it can be observed that the peak ratio of $\approx 10$ occurs at $v_{\mathrm{s}}=5 \mathrm{~km} \mathrm{~s}^{-1}$. To explain such behaviour, consider the $\mathrm{SO}$ abundance as a function of distance in Figs. 12 and 13 for a shock of $v_{\mathrm{s}}=5 \mathrm{~km} \mathrm{~s}^{-1}$ with density from $n_{\mathrm{H}}=10^{3} \mathrm{~cm}^{-3}$ and $n_{\mathrm{H}}=10^{6} \mathrm{~cm}^{-3}$.

Comparing the $v_{\mathrm{s}}=5 \mathrm{~km} \mathrm{~s}^{-1}$ and $n_{\mathrm{H}}=10^{3} \mathrm{~cm}^{-3}$ model in Fig. 11 with the abundance profile for the same initial conditions in Fig. 12 begins to explain the peak abundance ratio. It is clear that this arises as a result of the J-type shock injecting SO from the grain surface, whilst the C-type shock cannot sputter at these conditions. As $d$ approaches $10^{13} \mathrm{~cm}$ the $\mathrm{SO}$ abundance peaks at around $10^{-7}-$ an enhancement relative to the initial SO abundance of $\approx 100$. However, towards $d \approx 10^{13} \mathrm{~cm}$ the SO abundance drops off sharply as SO is destroyed. This destruction skews the average $\mathrm{SO}$ abundance, hence the peak abundance ratio in Fig. 11 being far smaller than the peak enhancement of 100 . Moreover, $T_{\max }$ of a C-type shock of $v_{\mathrm{s}}=5 \mathrm{~km} \mathrm{~s}^{-1}$ is $85 \mathrm{~K}$. Such a minimal change in $T$ through the shock is not sufficient to drive any significant gas-phase chemistry, hence the SO abundance remaining relatively constant throughout the shock in Fig. 12.

Additionally, as $v_{\mathrm{s}}$ increases the C-type shock sputtering becomes more effective whilst the J-type shock destroys SO at high $T$, resulting in the average post-shock abundance in a J-type 




Fig. 12. SO abundances for a shock with initial velocity $v_{\mathrm{s}}=5 \mathrm{~km} \mathrm{~s}^{-1}$ and density $n_{\mathrm{H}}=10^{3} \mathrm{~cm}^{-3}$.

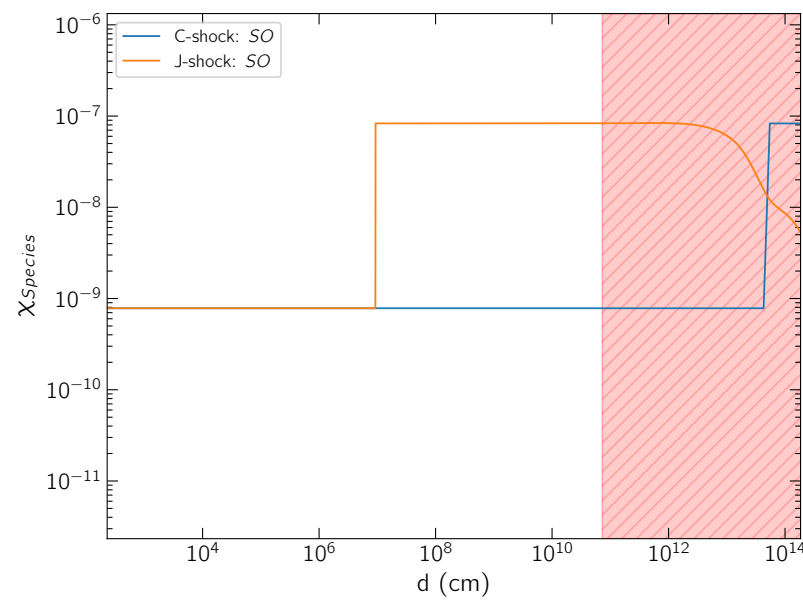

Fig. 13. SO abundances for a shock with initial velocity $v_{\mathrm{s}}=5 \mathrm{~km} \mathrm{~s}^{-1}$ and density $n_{\mathrm{H}}=10^{6} \mathrm{~cm}^{-3}$.

shock being less than the equivalent $\mathrm{C}$-type shock. For example at $v_{\mathrm{s}}=15 \mathrm{~km} \mathrm{~s}^{-1}$ and $n_{\mathrm{H}}=10^{3} \mathrm{~cm}^{-3}$, the J-type shock average abundance is $3 \times 10^{-2}$ times smaller than the C-type shock equivalent.

This is true of the models at $n_{\mathrm{H}}=10^{4} \mathrm{~cm}^{-3}$ as well, though here we note that the C-type shock sputtering is more efficient therefore exacerbating the differences between average abundance in shock type. Evident here is the J-type shock abundance at $v_{\mathrm{s}}=15 \mathrm{~km} \mathrm{~s}^{-1}$ and $n_{\mathrm{H}}=10^{4} \mathrm{~cm}^{-3}$ being $1 \times 10^{-2}$ times smaller than C-type shock equivalent.

Figures 12 and 13 also shows that as $n_{\mathrm{H}}$ increases, the abundances at large $d$ between shock types behaves universally and tends to a similar limit indicating that the dominant destruction mechanism becomes a density limited process. This therefore means that at lower $n_{\mathrm{H}}$, the enhancement is governed by a combination of gas-phase and dust-grain chemistry, whilst at large values of $n_{\mathrm{H}}$ the enhancement factor is governed by dust-grain chemistry alone.

\section{3. $\mathrm{SO}_{2}$}

Figure 14 shows the abundance ratios for $\mathrm{SO}_{2}$. Evident when considering Fig. 14 is the similarity between it and the SO behaviour in Fig. 11. Given that $\mathrm{SO}_{2}$ can form via $\mathrm{SO}$ dependent

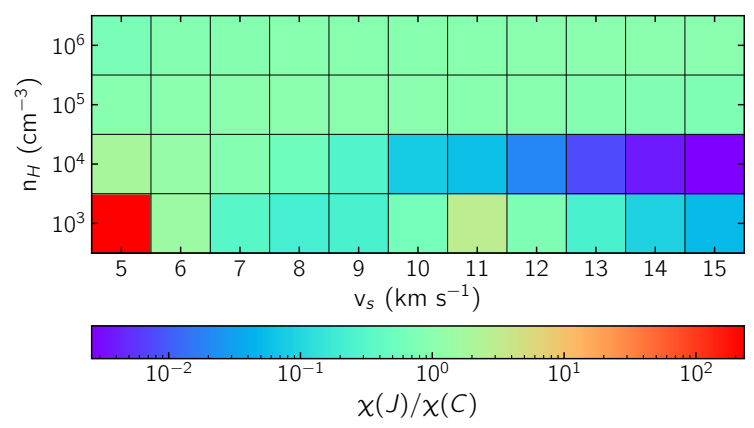

Fig. 14. Ratio of the maximum J-type enhanced $\mathrm{SO}_{2}$ abundance to the maximum C-type enhanced $\mathrm{SO}_{2}$ abundance. The largest difference between peak shock type abundance is at $n_{\mathrm{H}}=10^{3} \mathrm{~cm}^{-3}$ much like the SO abundance in Fig. 11.

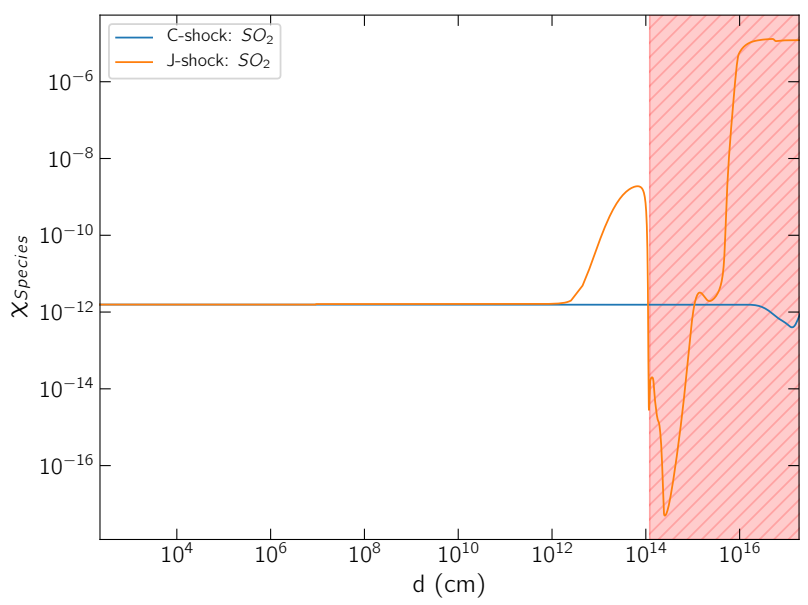

Fig. 15. $\mathrm{SO}_{2}$ abundances for a shock with initial velocity $v_{\mathrm{s}}=5 \mathrm{~km} \mathrm{~s}^{-1}$ and density of $n_{\mathrm{H}}=10^{3} \mathrm{~cm}^{-3}$.

reactions such as $\mathrm{O}+\mathrm{SO} \longrightarrow \mathrm{SO}_{2}$, the similarity in behaviour is not surprising.

Figure 14 shows largely the same trends as Fig. 11 did. For instance, we see the same behaviour in $\chi(J) / \chi(C) \approx 1$ at $n_{\mathrm{H}}>10^{4} \mathrm{~cm}^{-3}$ in Fig. 11, along with the same model having the same abundance ratio in Fig. 11. Curiously, this peak abundance ratio is $\approx 200$, whilst in Fig. 11 it was $\approx 10$. These global trends and behaviour are expected given the close chemical relationship between $\mathrm{SO}$ and $\mathrm{SO}_{2}$.

Figures 15 and 16 shows the $\mathrm{SO}_{2}$ abundances as a function of distance for both C-type and J-type shocks at $v=5 \mathrm{~km} \mathrm{~s}^{-1}$ through $n_{\mathrm{H}}=10^{3} \mathrm{~cm}^{-3}$ and $n_{\mathrm{H}}=10^{6} \mathrm{~cm}^{-3}$. Much like $\mathrm{SO}$ in Figs. 12 and 13, both C-type and J-type shock abundance tend to the same value as $n_{\mathrm{H}}$ increases. Furthermore the same behaviour is seen at low $n_{\mathrm{H}}$. This implies that any changes to SO in a shock should be mirrored - at least in terms of qualitative behaviour by $\mathrm{SO}_{2}$ as well.

\section{4. $\mathrm{HCN}$}

As Fig. 17 shows, the peak abundance ratio occurs at $v_{\mathrm{s}}<$ $9 \mathrm{~km} \mathrm{~s}^{-1}$ and $n_{\mathrm{H}}=10^{3} \mathrm{~cm}^{-3}$, with the degree of this ratio decreasing as $v_{\mathrm{s}}$ increases. As discussed before in Sects. 4.2.14.2.3 and 4.3 , it is the stark differences in sputtering and evaporation behaviour between shock types at these conditions that gives rise to this feature. 


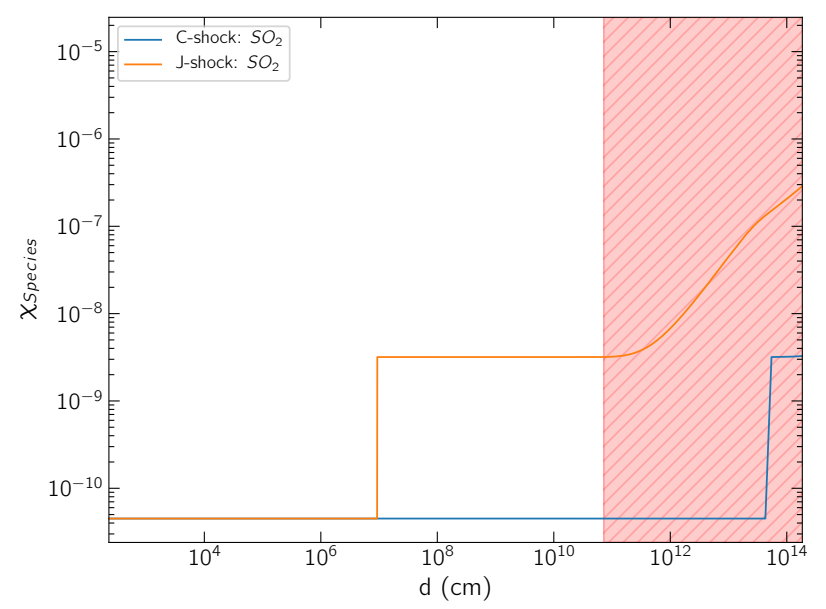

Fig. 16. $\mathrm{SO}_{2}$ abundances for a shock with initial velocity $v_{\mathrm{s}}=5 \mathrm{~km} \mathrm{~s}^{-1}$ and density of $n_{\mathrm{H}}=10^{6} \mathrm{~cm}^{-3}$.

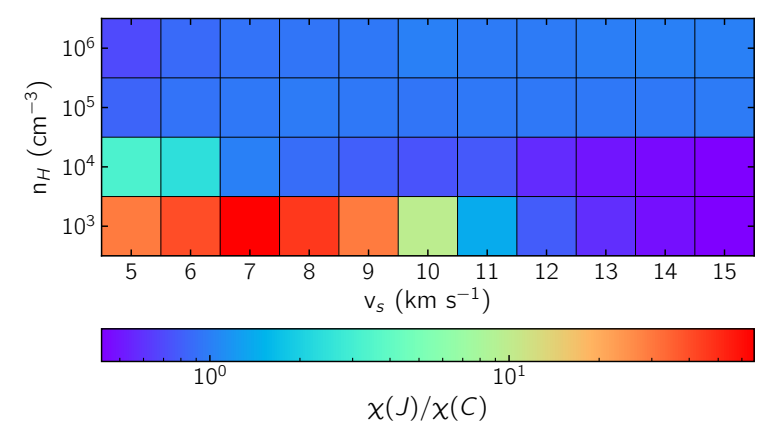

Fig. 17. Ratio of the average J-type enhanced HCN abundance to the average C-type enhanced $\mathrm{HCN}$ abundance. The largest difference between peak shock type abundance is at $v_{\mathrm{s}}<9 \mathrm{~km} \mathrm{~s}^{-1}$ and $n_{\mathrm{H}}=$ $10^{3} \mathrm{~cm}^{-3}$. High $v_{\mathrm{s}}$, low $n_{\mathrm{H}}$ shocks show C-type shocks are more efficient enhancers of HCN than equivalent J-type shocks.

Much like $\mathrm{SO}$ and $\mathrm{SO}_{2}$ beforehand, the ratio for $n_{\mathrm{H}}>$ $10^{4} \mathrm{~cm}^{-3}$ of Fig. 17 shows very little departure from 1 indicating that both shock types enhance $\mathrm{HCN}$ to the same or similar degree. Again similarly to $\mathrm{SO}$ and $\mathrm{SO}_{2}$ the enhancements at $v_{\mathrm{s}}=12-15 \mathrm{~km} \mathrm{~s}^{-1}$ and $n_{\mathrm{H}}=10^{3}-10^{4} \mathrm{~cm}^{-3}$ indicate C-type shocks are more effective enhancers of $\mathrm{HCN}$ than a J-type shock. As Table 2 shows, J-type shocks have far higher $T_{\max }$ than an equivalent C-type shock. This implies that between $v_{\mathrm{s}}=12-15 \mathrm{~km} \mathrm{~s}^{-1} \mathrm{~J}$-type shocks are capable of destroying HCN whilst an equivalent $\mathrm{C}$-type shock cannot reach a similarly high $T$, therefore allowing $\mathrm{HCN}$ to continue formation or not undergo destruction at all.

Individual abundance profiles for $\mathrm{HCN}$ are shown in Figs. 18 and 19. As is consistent with other figures, the immediate post-evaporation abundance increases as $n_{\mathrm{H}}$. Despite this, the maximal post-shock gas-phase enhancement of HCN is at lower density, with the effect dropping off as $n_{\mathrm{H}}$ increases.

Much like previous figures, Fig. 18 explains why the J-type shock HCN abundance is so much greater than the C-type shock HCN abundance. Similarly to before, C-type shock sputtering is not possible at $v_{\mathrm{s}}=5 \mathrm{~km} \mathrm{~s}^{-1}$ and $n_{\mathrm{H}}=10^{3} \mathrm{~cm}^{-3}$ whilst the J-type shock is capable of instantaneously evaporating the grain-mantle material. Unlike previous molecules however, this behaviour continues up to $v_{\mathrm{s}}=12 \mathrm{~km} \mathrm{~s}^{-1}$. As $n_{\mathrm{H}}$ increases to $n_{\mathrm{H}}=10^{6} \mathrm{~cm}^{-3}$ sputtering becomes more efficient and the postevaporation abundance increases no longer occur. Both of these



Fig. 18. $\mathrm{HCN}$ abundances for a shock with initial velocity $v_{\mathrm{s}}=5 \mathrm{~km} \mathrm{~s}^{-1}$ and density of $n_{\mathrm{H}}=10^{3} \mathrm{~cm}^{-3}$.

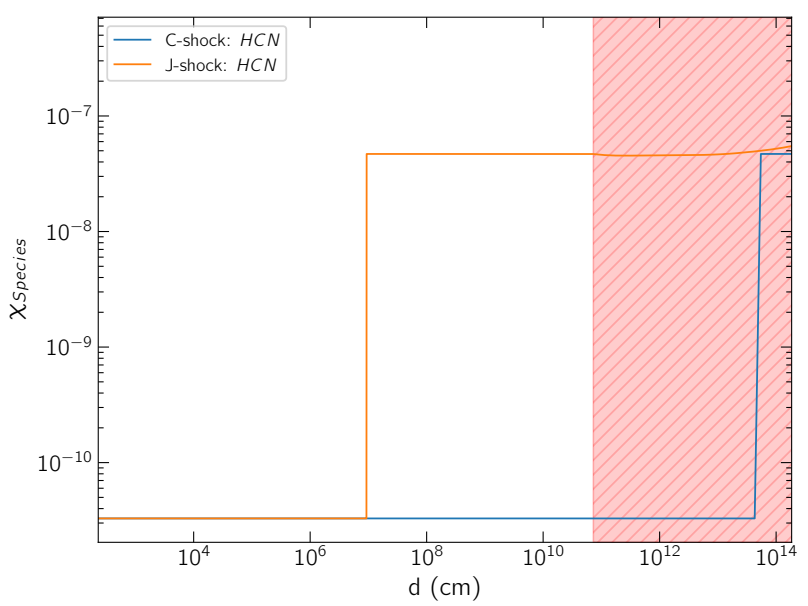

Fig. 19. $\mathrm{HCN}$ abundances for a shock with initial velocity $v_{\mathrm{s}}=5 \mathrm{~km} \mathrm{~s}^{-1}$ and density of $n_{\mathrm{H}}=10^{6} \mathrm{~cm}^{-3}$.

factors combined allows the $\mathrm{HCN}$ abundance in both shock types to tend to the same limit of $\approx 5 \times 10^{-8}$. As shown by Fig. 17, this behaviour occurs at all values of $v_{\mathrm{s}}$ for $n_{\mathrm{H}}=10^{5} \mathrm{~cm}^{-3}$ and $n_{\mathrm{H}}=10^{6} \mathrm{~cm}^{-3}$.

\section{The shocks in L1157-B2}

Vasta et al. (2012) observed $\mathrm{H}_{2} \mathrm{O}$ lines towards the B1 and B2 knots of L1157. In conjunction with theoretical shock models, they theorise that J-type shocks could be a prominent source of this emission. Consequently, having thus far found several unique J-type shock chemical distinctions, specifically with respect to $\mathrm{H}_{2} \mathrm{O}$ and $\mathrm{HCN}$, we qualitatively apply the results from our grid of models to the B2 region of L1157 in an effort to further categorise the type of shock responsible for its emission. We also compare the results to the measured abundances and enhancement factors in Table 1 to further constrain the shock type. Crucially, as mentioned in Sect. 2.2, the measured abundances are likely subject to large uncertainties owing to the optically thin and thermalised line assumptions required to determine them.

We focus on B2 and not B1 for a number of reasons. Firstly, Gusdorf et al. (2008) theorised that B1 is the result of a combination of C-type and J-type shocks, especially in regards to the 
$\mathrm{SiO}$ and $\mathrm{H}_{2}$ observations. This implies that $\mathrm{B} 2$ is also likely to be related to J-type shocks in some form. Further studies such as those by Vasta et al. (2012) also conclude that B2 likely hosts a J-type shock, either singularly or in combination with a C-type shock component. Lastly, given the low and high angular resolution observations of the B2 region by Benedettini et al. (2007, 2013), it seems that B2 is much more homogeneous than B1. This homogeneity removes any influence of successive shock driven chemistry, making B2 the ideal laboratory with which to test this type of shock diagnostic methodology.

\section{1. $\mathrm{CH}_{3} \mathrm{OH}$}

We showed in Sect. 4.2.1 that $\mathrm{CH}_{3} \mathrm{OH}$ undergoes no enhancement after its initial release from the dust grains into the gasphase. This therefore implies that $f(\mathrm{~B} 1)$ and $f(\mathrm{~B} 2)$ in Table 1 are dependent only upon the sputtered abundance and not the gas phase chemistry $\mathrm{CH}_{3} \mathrm{OH}$ undergoes.

According to Bachiller \& Pérez Gutiérrez (1997) L1157-B2 has a $\mathrm{CH}_{3} \mathrm{OH}$ abundance of $\approx 2.2 \times 10^{-5} \cdot \mathrm{CH}_{3} \mathrm{OH}$ 's minimal gasphase chemistry therefore means that shock enhancing $\mathrm{CH}_{3} \mathrm{OH}$ to this abundance is solely a result of sputtering and/or evaporation, which itself is a density-dependent effect. This implies that shock enhanced $\mathrm{CH}_{3} \mathrm{OH}$ traces the amount of $\mathrm{CH}_{3} \mathrm{OH}$ on the grains and therefore the density of the pre-shocked region, rather than the shock velocity.

According to Table 1 L1157-B2 has a $\mathrm{CH}_{3} \mathrm{OH}$ abundance 500 times larger than the central protostar, L1157-mm, where the $\chi_{\mathrm{CH}_{3} \mathrm{OH}}=4.5 \times 10^{-8}$. This is consistent with either a $\mathrm{C}$-type of J-type shock impacting a region of pre-shock density $n=10^{3} \mathrm{~cm}^{-3}$. This pre-shock density also produces a pre-shock abundance $\chi_{\mathrm{CH}_{3} \mathrm{OH}} \approx 2 \times 10^{-9}$, approximately consistent with the pre-shock density measured towards L1157-mm. Importantly this is also consistent with the pre-shock density reported by Gómez-Ruiz et al. (2016) towards L1157-B2. It remains difficult, however, to use $\mathrm{CH}_{3} \mathrm{OH}$ as a tracer of either shock type or shock velocity owing to its consistent gas-phase chemistry under differing physical conditions.

\section{2. $\mathrm{H}_{2} \mathrm{O}$}

We showed in Sect. 4.2.2 that $\mathrm{H}_{2} \mathrm{O}$ can trace J-type shocks at $v_{\mathrm{s}}<10 \mathrm{~km} \mathrm{~s}^{-1}$ and $n_{\mathrm{H}}=10^{3} \mathrm{~cm}^{-3}$.

In application to L1157-B2, however, no enhancement ratio was determined by Vasta et al. (2012). The $\mathrm{H}_{2} \mathrm{O}$ abundance towards L1157-B2 was determined as $1 \times 10^{-6}$. This abundance is smaller than all of the immediate post-evaporation/postsputtering abundances that our models show. These models can, however, recover an abundance similar to this for a shock of $v_{\mathrm{s}}<10 \mathrm{~km} \mathrm{~s}^{-1}$ and $n_{\mathrm{H}}=10^{3} \mathrm{~cm}^{-3}$. Matching the exact measured abundance is only achievable during the post-evaporation $\mathrm{H}_{2} \mathrm{O}$ abundance changes. At $v_{\mathrm{s}}>10 \mathrm{~km} \mathrm{~s}^{-1}, \mathrm{H}_{2} \mathrm{O}$ is destroyed in the gas-phase allowing the abundance to drop the order of $10^{-6}$, though as the temperature increases beyond that achieved in $v_{\mathrm{s}} \approx 12 \mathrm{~km} \mathrm{~s}^{-1}$ the abundance falls well below $10^{-6}$.

Importantly, the best matching shock conditions are also consistent with those determined by Gómez-Ruiz et al. (2016) as $v_{\mathrm{s}} \approx 10 \mathrm{~km} \mathrm{~s}^{-1}$ and $n_{\mathrm{H}} \approx 10^{3} \mathrm{~cm}^{-3}$. However, as we do not vary the freeze-out efficiency in this study we cannot conclude with certainty whether the observed abundance is solely a result of the shock or a combination of varying freeze-out efficiency and shock action. A lower freeze-out efficiency and slower shock velocity could reproduce a similar abundance to the observed abundance.

\section{3. $\mathrm{HCN}$}

We showed in Sect. 4.4 that HCN can undergo unique J-type shock enhancement at low $v_{\mathrm{s}}$ and low $n_{\mathrm{H}}$. As $v_{\mathrm{s}}$ and $n_{\mathrm{H}}$ increase the abundances in each shock type tend to a similar value, implying that $\mathrm{HCN}$ can trace low $v_{\mathrm{s}}$ and low $n_{\mathrm{H}} \mathrm{J}$-type shocks only.

Bachiller \& Pérez Gutiérrez (1997) estimate the HCN abundance towards L1157-B2 as $5.5 \times 10^{-7}$, undergoing an enhancement by a factor of $\approx 150$ relative to the L $1157-\mathrm{mm} \mathrm{HCN}$ abundance of $3.6 \times 10^{-9}$. Figures 18 and 19 shows both C-type and J-type shocks are capable of enhancing HCN to the same degree at high $n_{\mathrm{H}}$. Figures 18 and 19 also shows that whilst our models do not recover the exact initial HCN abundance of $3.6 \times 10^{-9}$ as measured towards L1157-mm, they are capable of re-producing a value of $\approx 10^{-9}$ in the range $n_{\mathrm{H}}=10^{3}-10^{5} \mathrm{~cm}^{-3}$.

Considering the enhancement factor of 150 , Fig. 17 shows that this is only possible in a J-type shock between $v_{\mathrm{s}}=6-8 \mathrm{~km} \mathrm{~s}^{-1}$ and $n_{\mathrm{H}}=10^{3} \mathrm{~cm}^{-3}$, which is approximately consistent with the shock parameters determined by Gómez-Ruiz et al. (2016).

\section{4. $S O$}

Bachiller \& Pérez Gutiérrez (1997) report that L1157-B2 is more abundant in $\mathrm{SO}$ than $\mathrm{SO}_{2}$. Crucially, the ranges defined for $\mathrm{SO}$ abundance in L1157-B1 and L1157-B2 by Bachiller \& Pérez Gutiérrez (1997) intersect, likely because of the close chemical relationship between $\mathrm{SO}$ and $\mathrm{SO}_{2}$.

The initial SO abundance measured towards L1157-mm is $5.0 \times 10^{-9}$. Much like HCN, our models are capable of recovering an initial SO abundance of $\approx 10^{-9}$ in the range $n_{\mathrm{H}}=$ $10^{3}-10^{5} \mathrm{~cm}^{-3}$.

Bachiller \& Pérez Gutiérrez (1997) measure the abundance of SO towards L1157-B2 as 2.0-5.0 $\times 10^{-7}$, which yields an enhancement ratio of 60-100. Our models, as is evident from Fig. 11, show that on average a J-type shock is not able to enhance SO to the same degree that an equivalent C-type can produce if both shock types can sputter and/or inject ice material into the gas-phase. This, therefore, implies that any unique SO enhancement is the result of a C-type shock.

\section{5. $\mathrm{SO}_{2}$}

According to Table 1, L1157-B2 is subject to an $\mathrm{SO}_{2}$ enhancement of $\approx 20$ with an initial abundance of $3.0 \times 10^{-8}$. None of the models produced here are capable of reproducing an initial abundance of this order. This indicates that $\mathrm{SO}_{2}$ has formed more efficiently towards L1157 than our models would indicate. In actuality, Table 1 shows $\mathrm{SO}_{2}$ being initially more abundant than SO by almost an order of magnitude.

Furthermore, the relative similarities in global behaviour between $\mathrm{SO}$ and $\mathrm{SO}_{2}$ mean the same conclusion applies here, i.e. within the grid of models, $\mathrm{C}$-type shocks are the producers of unique $\mathrm{SO}_{2}$ behaviour rather than J-type shocks.

\section{Discussion}

It is clear from Sect. 4 that the $\mathrm{CH}_{3} \mathrm{OH}, \mathrm{H}_{2} \mathrm{O}$ and $\mathrm{HCN}$ abundances do allude to a shock component within L1157-B2 of $v_{\mathrm{s}}=8-11 \mathrm{~km} \mathrm{~s}^{-1}$ impacting a region of pre-shock density of $n_{\mathrm{H}}=10^{3} \mathrm{~cm}^{-3}$. However, $\mathrm{CH}_{3} \mathrm{OH}$ does not undergo any gasphase enhancement unique to a specific shock type, rendering it a reliable tracer of pre-shock density in the majority of cases.

According to Figs. 6 and $7, \mathrm{CH}_{3} \mathrm{OH}$ undergoes no postevaporation gas-phase abundance change. Recent evidence 
(Holdship et al. 2019, and references therein) indicates that contrary to this finding, $\mathrm{CH}_{3} \mathrm{OH}$ is destroyed in highly energetic, high-temperature events such as shocks. The lack of $\mathrm{CH}_{3} \mathrm{OH}$ destruction in our models could indicate that the network used, in this instance UMIST, may be missing some of the dominant high-temperature destruction routes for $\mathrm{CH}_{3} \mathrm{OH}$. Alternatively, such findings could point to observations capturing a form of progressive erosion of $\mathrm{CH}_{3} \mathrm{OH}$ from the grain surfaces as first proposed by Jiménez-Serra et al. (2005). Nevertheless, there is sufficient evidence to consider the $\mathrm{CH}_{3} \mathrm{OH}$ abundances determined here as upper limits.

To address the apparent lack of destruction we performed a test whereby we included several combustion literature derived $\mathrm{CH}_{3} \mathrm{OH}$ destruction routes via collisional dissociation with $\mathrm{H}$ in our network. We selected these reactions as they are thought to be the most efficient mechanism for $\mathrm{CH}_{3} \mathrm{OH}$ destruction in dissociative J-type shocks (Suutarinen et al. 2014). Specifically, the reactions included are $\mathrm{CH}_{3} \mathrm{OH}+\mathrm{H} \longrightarrow \mathrm{CH}_{3}+\mathrm{H}_{2} \mathrm{O}$ (Hidaka et al. 1989), $\mathrm{CH}_{3} \mathrm{OH}+\mathrm{H} \longrightarrow \mathrm{H}_{2}+\mathrm{CH}_{2} \mathrm{OH}$ ( $\mathrm{Li} \&$ Williams 1996) and $\mathrm{CH}_{3} \mathrm{OH}+\mathrm{H} \longrightarrow \mathrm{H}_{2}+\mathrm{CH}_{3} \mathrm{O}$ (Warnatz 2012). However, the addition of these reactions produced complete destruction of gas-phase $\mathrm{CH}_{3} \mathrm{OH}$ at high temperature. This may be because the destruction reactions we included have only been measured under combustion conditions and hence are not necessarily accurate for the densities and temperatures of the ISM environments of our study (Balucani, priv. comm.). We therefore cannot draw any definitive conclusions regarding $\mathrm{CH}_{3} \mathrm{OH}$ abundance as a tracer of shock type, beyond the upper-limits derived here, until a follow-up study is performed to investigate the prominent reactions responsible for $\mathrm{CH}_{3} \mathrm{OH}$ 's high-temperature destruction. However, the injection behaviour of $\mathrm{CH}_{3} \mathrm{OH}$ provides an excellent tracer of pre-shock density.

$\mathrm{H}_{2} \mathrm{O}$ and $\mathrm{HCN}$ both exhibit degrees of enhancement that peak at a factor of 60 relative to a C-type shock at low $v_{\mathrm{S}}$ low $n_{\mathrm{H}}$. However the behaviour of both $\mathrm{HCN}$ and $\mathrm{H}_{2} \mathrm{O}$ at larger $n_{\mathrm{H}}$ tends to a common trend between both shock types. Such behaviour indicates that using $\mathrm{H}_{2} \mathrm{O}$ and $\mathrm{HCN}$ as shock type tracers is only valid, and likely only accurate, at lower values of $v_{\mathrm{s}}$ and $n_{\mathrm{H}}$.

Importantly we do not deplete the initial abundance of S. As Jenkins (2009) highlight, the observed abundance of $S^{+}$in earlystar forming regions matches the approximate cosmic abundance. However, as noted by Laas \& Caselli (2019), the abundance of S-bearing species in molecular clouds is reduced significantly, hence the term "depletion". Astrochemical models therefore tend to reduce the elemental S abundance to $1 \%$ of its cosmic abundance in order to ensure that the S-bearing molecular inventory is representative of the region studied, for example a molecular cloud. Given the uncertainty surrounding the exact depletion factors, both universally as well as locally, to introduce a depletion factor here would introduce another degree of freedom and another potentially significant source of error that may potentially yield a larger disagreement between the predictions and observations. We therefore fix our initial S abundance as solar.

Studies such as those by Benedettini et al. (2007) have shown the B1 and B2 knots themselves have sub-structure. These substructural features are likely not thermalised with their surroundings, rendering the observed molecular abundances more abundant than one discrete shock event would produce. It is also possible that B2 may host multiple velocity components, meaning that different molecules may trace different components of the shock. However, the upper limits of the $\mathrm{CH}_{3} \mathrm{OH}$ abundance for L1157-B2 derived here are consistent with a shock of pre-shock density $n_{\mathrm{H}}=10^{3} \mathrm{~cm}^{-3}$ which matches the pre-shock density determined by Gómez-Ruiz et al. (2016).
Gómez-Ruiz et al. (2016) use $\mathrm{NH}_{3}$ and $\mathrm{H}_{2} \mathrm{O}$ observations to derive their estimates of the shock parameters. Our $\mathrm{H}_{2} \mathrm{O}$ abundance trends show an immediate post-shock enhanced abundance of between $10^{-4}$ and $10^{-5}$, around a factor 10 to $10^{2}$ higher than Vasta et al. (2012) would indicate. As mentioned previously, some models are capable of achieving an $\mathrm{H}_{2} \mathrm{O}$ abundance approximately consistent with observations providing that the shock is capable of dissociating $\mathrm{H}_{2} \mathrm{O}$. Consequently, the observations could be tracing this dissociated $\mathrm{H}_{2} \mathrm{O}$ component. Alternatively, our simulations may over-estimate the formation efficiency of $\mathrm{H}_{2} \mathrm{O}$ on the grains, thus allowing more $\mathrm{H}_{2} \mathrm{O}$ to be released from the grains in sputtering or evaporation than is realistic.

From Table 1 the abundance of $\mathrm{H}_{2} \mathrm{O}$ in $\mathrm{L} 1157$ is around 2 orders of magnitude higher in $\mathrm{B} 1$ than $\mathrm{B} 2$, indicating that gasphase $\mathrm{H}_{2} \mathrm{O}$ has either been destroyed in L1157-B2 or that it has had sufficient time to freeze on to the surface of the dust.

The behaviour of $\mathrm{SO}$ and $\mathrm{SO}_{2}$ in Figs. 11 and 14 show that they both reliably trace low $v_{\mathrm{S}}$, low $n_{\mathrm{H}} \mathrm{C}$-type shocks. We have thus far established that L1157-B2 bears some signatures of J-type shock chemistry, but $\mathrm{SO}$ and $\mathrm{SO}_{2}$ trace predominantly $\mathrm{C}$-type shocks in the parameter space considered. However, the $\mathrm{SO}$ and $\mathrm{SO}_{2}$ behaviour, coupled with the $\mathrm{SO}$ and $\mathrm{SO}_{2}$ enhancements in L1157-B2, would seemingly imply that L1157-B2 is host to either a C-type shock component as well as a J-type shock component or a singular component being mixed-type in nature. This mixed-type shock could potentially be a J-type shock that is evolving to take on a more C-type shock structure. Both of these scenarios are consistent with the observed trends. However, to confirm either scenario would require a more detailed model of a mixed-type shock, though observations such as those by Benedettini et al. $(2007,2013)$ are sufficient to continue exploring this question.

Further observational constraints will surely also be provided by SOLIS (Ceccarelli et al. 2017). Such data may allow classification of whether B2 hosts any sub-structure, in turn informing even further constraints on theoretical models of shock action.

\section{Summary}

We have developed a parameterised model of an isothermal, planar J-type shock wave as a module to the astrochemical code UCLCHEM. We compute a grid of models across the parameter space $v_{\mathrm{s}}=5-15 \mathrm{~km} \mathrm{~s}^{-1}$ and $n_{\mathrm{H}}=10^{3}-10^{6} \mathrm{~cm}^{-3}$ using our J-type shock model as well as the pre-existing C-type module to quantify the different chemical abundance trends in each shock type. We find the following.

1. Our results show that whilst a theoretical distinction in J-type shock chemistry is found in molecules such as $\mathrm{H}_{2} \mathrm{O}$ and $\mathrm{HCN}$, it is largely unique to low $v_{\mathrm{s}}$, low $n_{\mathrm{H}}$ shocks owing to the extreme temperatures J-type shocks are capable of reaching at high values of $v_{\mathrm{s}}$. Furthermore, the largest differences in chemistry between shock types arises as a result of the different sputtering and evaporation behaviours between shock types at low $v_{\mathrm{s}}$ and low $n_{\mathrm{H}}$.

2. We find that $\mathrm{CH}_{3} \mathrm{OH}$ is enhanced by shocks and is a reliable probe of the pre-shock gas density, however we find no difference between its gas-phase abundance between shock type. Recent evidence (Holdship et al. 2019) indicates that $\mathrm{CH}_{3} \mathrm{OH}$ is destroyed in high $T$ shocks, indicating that chemical networks lack the high $T$ reactions that permit $\mathrm{CH}_{3} \mathrm{OH}$ to be destroyed. Consequently, astrochemical simulations such as the one presented here can only provide upper limits of the shock-enhanced $\mathrm{CH}_{3} \mathrm{OH}$ abundance. 
3. Finally in our application to the L1157-B2 region, we find that fractional abundances are consistent with both C-type and J-type shock emission, potentially indicating the prevalence a mixed-type shock or multiple shock components. Crucially, however, the similarities in abundances at the initial conditions considered here indicate that the dominant factors affecting shock chemistry are more dependent on the initial shock conditions and not the shock type.

Acknowledgements. T. A. J. is funded by an STFC studentship, and thanks the STFC accordingly. I. J.-S. has received partial support from the Spanish FEDER (project number ESP2017-86582-C4-1-R), and State Research Agency (AEI) through project number MDM-2017-0737 Unidad de Excelencia "María de Maeztu" - Centro de Astrobiología (INTA-CSIC). The authors would like to thank the anonymous referee for their very valuable comments that helped improve the manuscript. We also thank D. Williams, R. Garrod, and N. Balucani for their discussions and opinions on aspects of this paper.

\section{References}

Allen, M., \& Robinson, G. W. 1977, AJ, 212, 396

Bachiller, R., \& Pérez Gutiérrez, M. 1997, ApJ, 487, L93

Bachiller, R., Perez-Gutierrez, M., Kumar, M. S. N., \& Tafalla, M. 2001, A\&A, 372, 899

Baulch, D. L., Pilling, M. J., Cobos, C. J., et al. 1992, J. Phys. Chem. Ref. Data, 21, 411

Benedettini, M., Viti, S., Codella, C., et al. 2007, MNRAS, 381, 1127

Benedettini, M., Busquet, G., Lefloch, B., et al. 2012, A\&A, 539, L3

Benedettini, M., Viti, S., Codella, C., et al. 2013, MNRAS, 436, 179

Ceccarelli, C., Caselli, P., Fontani, F., et al. 2017, ApJ, 850, 176

Charnley, S. B. 1997, ApJ, 481, 396

Chièze, J. P., Des ForÉTs, G. P., \& Flower, D. R. 1998, MNRAS, 295, 672

Draine, B. T. 1980, ApJ, 241, 1021

Draine, B. T., Roberge, W. G., \& Dalgarno, A. 1983, ApJ, 264, 485

Flower, D. R., \& Des ForÉTs, G. P 2015, A\&A, 578, A63

Flower, D. R., Le Bourlot, J., Des ForÉTs, G. P., \& Cabrit, S. 2003a, Space Sci. 287,183

Flower, D. R., Bourlot, J. L., Des ForÉTs, G. P., \& Cabrit, S. 2003b, MNRAS, 80,70

Fraser, H. J., Collings, M. P., McCoustra, M. R., \& Williams, D. A. 2001, MNRAS, 327, 1165

Fuchs, G. W., Cuppen, H. M., Ioppolo, S., et al. 2009, A\&A, 505, 629

Garrod, R. T., Weaver, S. L. W., \& Herbst, E. 2008, ApJ, 682, 283

Gidalevich, E. Y. 1966, Astrofizika, 02, 1966

Godard, B., Des ForÉTs, G. P., Lesaffre, P., et al. 2019, A\&A, 622, A208
Gómez-Ruiz, A. I., Codella, C., Viti, S., et al. 2016, MNRAS, 462, 2203

Gueth, F., Guilloteau, S., \& Bachiller, R. 1996, A\&A, 307, 891

Gusdorf, A., Des ForÉTs, G. P., Cabrit, S., \& Flower, D. R. 2008, A\&A, 490, 695

Hidaka, Y., Oki, T., Kawano, H., \& Higashihara, T. 1989, J. Phys. Chem., 93 7134

Holdship, J., Viti, S., Jiménez-Serra, I., Makrymallis, A., \& Priestley, F. 2017, AJ, 154, 38

Holdship, J., Viti, S., Codella, C., et al. 2019, AJ, 880, 138

Hollenbach, D., \& McKee, C. F. 1989, ApJ, 342, 306

Hugoniot, H. 1889, J. 1'Ecole Polytech., 58, 1

Jenkins, E. B. 2009, ApJ, 700, 1299

Jiménez-Serra, I., Caselli, P., Martin-Pintado, J., \& Hartquist, T. W. 2008, A\&A, 482,549

Jiménez-Serra, I., Martín-Pintado, J., Rodríguez-Franco, A., \& Martín, S. 2005, ApJ, 627, L121

Jiménez-Serra, I., Caselli, P., Martín-Pintado, J., \& Hartquist, T. W. 2008, A\&A, 482, 549

Laas, J. C., \& Caselli, P. 2019, A\&A, 624, A108

Lefloch, B., Cabrit, S., Codella, C., et al. 2010, A\&A, 518, L113

Lefloch, B., Bachiller, R., Ceccarelli, C., et al. 2018, MNRAS, 477, 4792

Li, S. C., \& Williams, F. A. 1996, Symp. (Int.) Combust., 26, 1017

Looney, L. W., Tobin, J. J., \& Kwon, W. 2007, ApJ, 670, L131

Martinez, A. P. 2009, Astron. J., 2, 1

McElroy, D., Walsh, C., Markwick, A. J., et al. 2013, A\&A, 550, A36

Podio, L., Codella, C., Gueth, F., et al. 2016, A\&A, 593, L4

Rankine, W. J. M. 1870, Philos. Trans. R. Soc. London, 160, 277

Rawlings, J. M. C., Hartquist, T. W., Menten, K. M., \& Williams, D. A. 1992, MNRAS, 255, 471

Rodríguez-Fernández, N. J., Tafalla, M., Gueth, F., \& Bachiller, R. 2010, A\&A, 516, A98

Shu, F. H., Ruden, S. P., Lada, C. J., \& Lizano, S. 1991, ApJ, 370, L31

Snell, R. L., Loren, R. B., \& Plambeck, R. L. 1980, ApJ, 239, L17

Suutarinen, A. N., Kristensen, L. E., Mottram, J. C., Fraser, H. J., \& Van Dishoeck, E. F. 2014, MNRAS, 440, 1844

Tafalla, M., \& Bachiller, R. 1995, ApJ, 443, L37

Tielens, A. G. G. M., \& Whittet, D. C. B. 1997, Molecules in Astrophysics: Probes \& Processes: Abstract Book, 178, 45

Umemoto, T., Iwata, T., Fukui, Y., et al. 1992, ApJ, 392, L83

van Dishoeck, E. F., Kristensen, L. E., Benz, A. O., et al. 2010, PASP, 123, 138

van Dishoeck, E. F., Herbst, E., \& Neufeld, D. A. 2013, Chem. Rev., 113, 9043

Vasta, M., Codella, C., Lorenzani, A., et al. 2012, A\&A, 537, A98

Viti, S., Jiménez-Serra, I., Yates, J. A., et al. 2011, ApJ, 740, L3

Warnatz, J. 2012, Combustion Chemistry (New York: Springer), 197

Williams, D. A., \& Viti, S. 2013, Observational Molecular Astronomy (Cambridge: Cambridge University Press)

Zel'dovich, Y. B., \& Raizer, Y. P. 1967, Physics of Shock Waves and Hightemperature Hydrodynamic Phenomena (New York: Academic Press)

Zhang, Q., \& Zheng, X. 1997, ApJ, 474, L719 